\title{
FINITELY DECIDABLE CONGRUENCE MODULAR VARIETIES
}

\author{
JOOHEE JEONG
}

\begin{abstract}
A class $\mathscr{V}$ of algebras of the same type is said to be finitely decidable iff the first order theory of the class of finite members of $\mathscr{V}$ is decidable. Let $\mathscr{V}$ be a congruence modular variety. In this paper we prove that if $\mathscr{V}$ is finitely decidable, then the following hold. (1) Each finitely generated subvariety of $\mathscr{V}$ has a finite bound on the cardinality of its subdirectly irreducible members. (2) Solvable congruences in any locally finite member of $\mathscr{V}$ are abelian. In addition we obtain various necessary conditions on the congruence lattices of finite subdirectly irreducible algebras in $\mathscr{V}$.
\end{abstract}

\section{INTRODUCTION}

The study of the decidability of first order theories has been one of the major themes in mathematical logic since the beginning of the 20th century. In earlier days, research in the theory of decidability was focused on determining whether a particular theory is decidable. These days, after the pioneering works of Zamyatin [22-25] and Burris and McKenzie [2], higher level questions like "when is a theory decidable?" are being asked and (partially) answered [3, 8$10,13,17,19-21]$.

The problem of characterizing finitely decidable varieties was raised in various places such as $[2,4,17]$. Recently in the late 1980 s, Idziak was able to completely characterize the finite decidability for those varieties which are congruence distributive, finitely generated and of finite type [8-10]. This paper is an attempt to extend his result in the direction of replacing "distributive" by "modular".

It turned out that finitely decidable and congruence modular varieties have very nice structural properties. For instance, if such a variety is finitely generated and of finite type, then there exist only finitely many subdirectly irreducible algebras, which are all finite.

For notations in general, we followed those in $[6,7,16]$. We will be using a few more notations which are as follows. A subdirect power D of A (to a set, say $X$ ) is said to be diagonal iff $D$ contains all the constant maps. We write $\mathbf{D} \leq_{\text {diag }} \mathbf{A}^{X}$ in this situation. If $D \subseteq A^{X}$, and $B \subseteq A$, then $D(B)$ denotes the set $D \cap B^{X}$. If $f, g \in A^{X}$ and $\phi$ is a binary relation on $A$, then the $\phi$-equalizer of $f$ and $g$ is a subset of $X$ given by

$$
\llbracket\langle f, g\rangle \in \phi \mathbb{\prod} \stackrel{\text { def }}{=}\{x \in X \mid\langle f(x), g(x)\rangle \in \phi\} .
$$

Received by the editors June 17, 1991.

1991 Mathematics Subject Classification. Primary 08A05. 
$D(\phi)$ is defined to be the binary relation on $D$ such that

$$
\langle f, g\rangle \in D(\phi) \Leftrightarrow \mathbb{L}\langle f, g\rangle \in \phi \mathbb{\rrbracket}=X .
$$

$D \subseteq A^{X}$ is said to be $\phi$-closed iff for all $f \in A^{X}$ and $g \in D,\langle g, f\rangle \in D(\phi)$ implies $f \in D$. A finite Boolean triple is a first order structure $\left\langle B, B_{0}, B_{1}, \leq\right\rangle$, where $\langle B, \leq\rangle$ is a finite Boolean lattice order and $B_{i} \quad(i<2)$ are subuniverses of the associated Boolean algebra $\mathbf{B}$. The class of all finite Boolean triples is hereditarily undecidable. (The proof can be found, for instance, in [9].)

\section{Preliminary}

Throughout this paper, we fix a variety $\mathscr{V}$ which is finitely decidable and congruence modular. Then, by (8.5) and (11.1) of [7], every prime quotient in a finite algebra in $\mathscr{V}$ has type either 2 or 3 and has minimal sets with empty tails. We will be making use of this fact frequently throughout this paper without further notice. One more fact about our variety $\mathscr{V}$, which will be very useful in the arguments in this paper, is that whenever $\mathbf{A}$ is finite member of $\mathscr{V}$ there exists a ternary term $p(x, y, z)$ such that $\mathbf{A} \vDash p(x, y, y) \approx x \approx p(y, y, x)$ [13]. It is well known that the existence of such a term $p(x, y, z)$, which is called a Maltsev term for $\mathbf{A}$, implies, for all $a, b, c, d \in A$,

$$
\langle a, b\rangle \in \mathrm{Cg}^{\mathbf{A}}(c, d) \Leftrightarrow\left(\exists \lambda \in \operatorname{Pol}_{1} \mathbf{A}\right)(\langle\lambda(c), \lambda(d)\rangle=\langle a, b\rangle) .
$$

Let $\mathbf{A}$ be any algebra in $\mathscr{V}$. Then, for all $x, y, u, v \in A$, we have the following bi-implications:

$$
\begin{aligned}
\langle x, y\rangle & \in\left(0_{A}: \mathrm{Cg}^{\mathbf{A}}(u, v)\right) \\
& \Leftrightarrow \mathrm{Cg}^{\mathbf{A}}(x, y) \leq\left(0_{A}: \mathrm{Cg}^{\mathbf{A}}(u, v)\right) \\
& \Leftrightarrow \mathrm{Cg}^{\mathbf{A}}(u, v) \leq\left(0_{A}: \mathrm{Cg}^{\mathbf{A}}(x, y)\right) \\
& \Leftrightarrow\langle u, v\rangle \in\left(0_{A}: \mathrm{Cg}^{\mathbf{A}}(x, y)\right) .
\end{aligned}
$$

Assume that $\mathbf{A}$ is finite. Then, for each $a, b \in A$, there exists a unary polynomial $\lambda(x)$ of $\mathbf{A}$ that switches $a$ and $b$; for instance, the polynomial $\lambda(x) \stackrel{\text { def }}{=} p(a, x, b)$, where $p$ is a Maltsev term for $\mathbf{A}$, is one such polynomial. Combining this with (1) and (2), we get the following: for all $x, y, u, v \in A$,

$$
\begin{aligned}
\langle x, y\rangle \in & \left(0_{A}: \mathrm{Cg}^{\mathbf{A}}(u, v)\right) \\
& \Leftrightarrow\left(\forall s \in \mathrm{Pol}_{2} \mathbf{A}\right)(s(x, u)=s(x, v) \Rightarrow s(y, u)=s(y, v)) \\
& \Leftrightarrow\left(\forall s \in \mathrm{Pol}_{2} \mathbf{A}\right)(s(x, v)=s(y, v) \Rightarrow s(x, u)=s(y, u)) .
\end{aligned}
$$

Now suppose that $x, y, u, v, a \in A, s \in \operatorname{Pol}_{2} \mathbf{A}$, and $\langle x, y\rangle \in\left(0_{A}: \mathrm{Cg}^{\mathbf{A}}(u, v)\right)$. Then

$$
p(s(x, u), s(x, \check{u}), a)=a=p(s(y, u), s(y, \check{u}), a) .
$$

In this equality, we can replace the $u$ 's marked by "“" by $v$ 's since $\langle u, v\rangle \in$ $\left(0_{A}: \mathrm{Cg}^{\mathbf{A}}(x, y)\right)$, and get

$$
p(s(x, u), s(x, v), a)=p(s(y, u), s(y, v), a) .
$$

Conversely, suppose that (4) holds for all $s \in \operatorname{Pol}_{2} \mathbf{A}, a \in A$. Then when we assume $s(x, v)=s(y, v)$, we obtain $s(x, u)=s(y, u)$ by replacing $a$ 's in (4) 
by $s(x, v)$ 's (or equivalently, by $s(y, v)$ 's). Now by (3), we get (5)

$$
\begin{aligned}
& \langle x, y\rangle \in\left(0_{A}: \mathrm{Cg}^{\mathbf{A}}(u, v)\right) \\
& \quad \Leftrightarrow\left(\forall s \in \mathrm{Pol}_{2} \mathbf{A}, a \in A\right)(p(s(x, u), s(x, v), a)=p(s(y, u), s(y, v), a)) .
\end{aligned}
$$

Let $\theta \in$ Con $\mathbf{A}$ be given and let $x, y, u, v \in A$. Then $\langle x, y\rangle \in\left(0_{A}: \theta\right)$ iff $\langle x, y\rangle \in\left(0_{A}: \operatorname{Cg}^{\mathbf{A}}(u, v)\right)$ for all $\langle u, v\rangle \in \theta$. Thus, by combining (3) and (5), we get

$$
\begin{aligned}
& \langle x, y\rangle \in\left(0_{A}: \theta\right) \\
& \Leftrightarrow\left(\forall s \in \mathrm{Pol}_{2} \mathbf{A},\langle u, v\rangle \in \theta\right)(s(x, u)=s(x, v) \Rightarrow s(y, u)=s(y, v)) \\
& \Leftrightarrow\left(\forall s \in \mathrm{Pol}_{2} \mathbf{A},\langle u, v\rangle \in \theta\right)(s(x, u)=s(y, v) \Rightarrow s(x, u)=s(y, u)) \\
& \Leftrightarrow\left(\forall s \in \mathrm{Pol}_{2} \mathbf{A},\langle u, v\rangle \in \theta, a \in A\right)(p(s(x, u), s(x, v), a)) \\
& =p(s(y, u), s(y, v), a)) .
\end{aligned}
$$

Thus we see that whenever $\mathbf{D} \leq_{\operatorname{diag}} \mathbf{A}^{\mathbf{X}}$ and $\alpha \in \operatorname{Con} \mathbf{A}$ is a centralizer of some congruence of $\mathbf{A}$, the binary predicate $\langle f, g\rangle \in D(\alpha)$ (or equivalently, $\llbracket\langle f, g\rangle \in \alpha \rrbracket=X)$ on $D$ is first order definable in $\mathbf{D}$ by (7).

One tame congruence theoretic fact that we will be using frequently in this paper is that if $U$ is a minimal set relative to some prime quotient in a finite algebra $\mathbf{A}$, then whenever $\mathbf{D}$ is a diagonal subdirect power of $\mathbf{A}, D(U)$ is definable in $\mathbf{D}$ by a first order formula; to prove this, choose an idempotent unary polynomial $e$ of $\mathbf{A}$ so that $e(A)=U$, and note that

$$
(\forall f \in D)(f \in D(U) \Leftrightarrow e(f)=f) .
$$

\section{NONABELIAN MONOLITH CASE}

In this section, we will investigate the finite subdirectly irreducible members of $\mathscr{V}$ with nonabelian monoliths.

Lemma 1. Let $\mathbf{A}$ be a finite subdirectly irreducible algebra in $\mathscr{V}$ with a monolith of type 3. Then, for any $a \neq b \in A$, there exists a first order formula $\sigma(x)$ such that whenever $\mathbf{D}$ is a diagonal subdirect power of $\mathbf{A}$, we have

$$
(\forall f \in D)(f \in D(\{a, b\}) \Leftrightarrow \mathbf{D} \vDash \sigma(f)) .
$$

Proof. The proof is found in [13].

Theorem 2. Let $\mathbf{A}$ be a finite subdirectly irreducible member of $\mathscr{V}$ with a nonabelian monolith. Then $\operatorname{typ}\{\mathbf{A}\}=\{\mathbf{3}\}$.

Proof. Let $\mu$ be a monolith of $\mathbf{A}$. Then the type of $\mu$ is $\mathbf{3}$ and hence we can use Lemma 1. Let $N$ be a $\left\langle 0_{A}, \mu\right\rangle$-minimal set, say $N=\{0,1\}$. Then clearly $\mathrm{Cg}^{\mathbf{A}}(0,1)=\mu$. Assume, on the contrary, that there exists a prime quotient $\langle\delta, \theta\rangle$ in Con $\mathrm{A}$ with type not equal to 3 . We will get a contradiction by interpreting the class of all finite graphs into $\operatorname{Sp}_{\mathrm{fin}}(\mathbf{A})$.

By tame congruence theory, we know that $\operatorname{typ}(\delta, \theta)=2$. Choose a $\langle\delta, \theta\rangle$ minimal set $U$ and choose an ordered pair $\langle a, b\rangle$ in a trace of $U$ so that $\langle a, b\rangle \notin \delta$. Let a finite graph $\mathbf{G} \stackrel{\text { def }}{=}\langle V, E\rangle$ be given. Wlog $V \cap E=\varnothing$. Pick $x_{0}$ not in $V \cup E$ and let $X=\left\{x_{0}\right\} \cup V \cup E$. Let $v_{0}, v_{1}, \ldots, v_{n-1}$ be a 
one-to-one enumeration of $V$. For each $i<n$, let $h_{i} \in\{a, b\}^{X}$ be defined by

$$
\begin{aligned}
h_{i}(x) & =b & & \text { if } x=v_{i} \text { or } v_{i} \in x \in E, \\
& =a & & \text { otherwise. }
\end{aligned}
$$

Let $H=\left\{h_{i} \mid i<n\right\}$. Then it is obvious that $H$ is in one-to-one correspondence with $V$ via the map $h_{i} \mapsto v_{i}$. Let $\mathrm{D}$ be the subalgebra of $\mathbf{A}^{X}$ generated by $H \cup\{\hat{a}: a \in A\}$. Note that $\mathbf{D}$ is a diagonal subdirect power of $\mathbf{A}$ and hence $D(\{a, b\})$ is first order definable in $\mathbf{D}$ by Lemma 1 .

By (1) and basic tame congruence theory, we get a unary polynomial $\lambda(x)$ of A such that $\langle\lambda(a), \lambda(b)\rangle=\langle 0,1\rangle$ and $\operatorname{ran}(\lambda)=\{0,1\}$. If we let $\operatorname{meet}(x, y)$ be a binary polynomial operation of $\mathbf{A}$ such that meet $\left.\right|_{N}$ is the Boolean meet operation of $\left.\mathbf{A}\right|_{N}$, and let a formula $\operatorname{Edge}(x, y)$ be given by

$$
\operatorname{Edge}(x, y)=(\operatorname{meet}(\lambda(x), \lambda(y)) \not \approx 0) \wedge(x \not \approx y),
$$

then for $h_{i}, h_{j} \in H$, we have

$$
\left\{v_{i}, v_{j}\right\} \in E \Leftrightarrow \mathbf{D} \vDash \operatorname{Edge}\left(h_{i}, h_{j}\right) .
$$

It remains to find a first order formula that defines $H$ in $\mathbf{D}$. Let $h^{0}, h_{V} \in$ $D(N)$ be defined by

$$
h^{0}(x)=0 \quad \text { iff } \quad x=x_{0}, \quad h_{V}(x)=1 \quad \text { iff } \quad x \in V,
$$

and let $\phi(x)$ be a formula saying that

$$
\begin{aligned}
& x \in D(\{a, b\}) \& \lambda(x) \vee h^{0} \not 1 \& \lambda(x) \wedge h_{V} \not \hat{0} \& \\
& \quad(\forall y \in D)\left(\left(y \in D(\{0,1\}) \& y \wedge h_{V}<\lambda(x) \wedge h_{V}\right) \Rightarrow y \wedge h_{V} \approx \hat{0}\right) .
\end{aligned}
$$

Here we are using the fact that there exist binary polynomial operations of $\mathbf{A}$ which are the Boolean meet and join operations in $\left.\mathbf{A}\right|_{N}$. Also note that $h^{0}$ and $h_{V}$ are in fact members of $D$ since $h^{0}=\bigvee\{\lambda(h) \mid h \in H\}$ and $h_{V}=h^{0}-$ $\bigvee\left\{\lambda\left(h_{i}\right) \wedge \lambda\left(h_{j}\right) \mid\left\{v_{i}, v_{j}\right\} \in E\right\}$, where " - " is the Boolean difference operation on $\left.\mathbf{A}\right|_{N}$. We claim that $\phi$ defines $H$ in $\mathbf{D}$. Indeed each $h_{i} \in H$ is easily seen to satisfy $\phi$. It remains to show that $\mathbf{D} \vDash \phi(f) \Rightarrow f \in H$ for all $f \in D$.

Assume that $f \in D$ and $\mathbf{D} \vDash \phi(f)$. We want to show $f \in H$. First of all, $f$ is a member of $D$, which is generated in $\mathbf{A}^{X}$ by members of $H$ and the constant maps. So we can find a polynomial operation $q(\bar{x})$ with arity $n$ such that $f=q\left(h_{0}, \ldots, h_{n-1}\right)$. By applying the idempotent unary polynomial of A whose range is $U$ to both sides of this identity, we may assume that $\operatorname{ran}(q) \subseteq U$. Moreover, $q(\bar{a})=q\left(h_{0}, \ldots, h_{n-1}\right)\left(x_{0}\right)=f\left(x_{0}\right)=a$, where the last equality $\left(x_{0}\right)=a$ comes from $\lambda(f) \vee h_{0} \neq \hat{1}$. Thus $q(a / \theta \cap U) \subseteq a / \theta \cap U$, which means we may assume that $q$ is a polynomial of the induced algebra $\left.\mathbf{A}\right|_{a / \theta \cap U}$. If we let $M=(a / \theta \cap U) / \delta$, then $\left.(\mathbf{A} / \delta)\right|_{M}$ is polynomially equivalent to a module $M$ over a ring, say $R$. Further, we can assume that $a / \delta$ is the 0 -element of the module. Choose $c / \delta \in M$ and $r_{0}, \ldots, r_{n-1} \in R$ so that $f / \delta=q(\bar{h}) / \delta=\sum_{i<n} r_{i} \cdot\left(h_{i} / \delta\right)+c / \delta$. Let us look at the value of each side of this identity at $x_{0}$. We already know that $f\left(x_{0}\right)=a$, and thus $f\left(x_{0}\right) / \delta=a / \delta=$ $0^{M}$. But the value of the r.h.s. at $x_{0}$ is just $c / \delta$ since $h_{i}\left(x_{0}\right) / \delta=a / \delta=0^{M}$ for all $i<n$. Thus $c / \delta=0^{M}$ and we can now write

$$
\frac{f}{\delta}=\sum_{i<n} r_{i} \cdot\left(\frac{h_{i}}{\delta}\right)
$$


$\lambda(f) \wedge h_{V} \neq \hat{0}$, which is one of the conjuncts of $\phi(f)$, implies that there exists at least one $j<n$ such that $f\left(v_{j}\right)=b$, and the last conjunct of $\phi(f)$ tells us that there exists at most one $j<n$ for which $f\left(v_{j}\right)=b$. Choose the unique $j<n$ so that $f\left(v_{j}\right)=b$ and call it $k$. Let $j<n$ be given. Then by evaluating the identity (8) at $v_{j}$, we have $\left(v_{j}\right) / \delta=\sum_{i<n} r_{i} \cdot\left(h_{i}\left(v_{j}\right) / \delta\right)=r_{j} \cdot(b / \delta)$. So we have

$$
\begin{aligned}
r_{j} \cdot(b / \delta) & =b / \delta & & \text { if } j=k, \\
& =a / \delta & & \text { otherwise. }
\end{aligned}
$$

But for $r \in R$ and $h \in D(\{a, b\}), r \cdot(b / \delta)=a / \delta$ implies $r \cdot(h / \delta)=\hat{a} / \delta$ and $r \cdot(b / \delta)=b / \delta$ implies $r \cdot(h / \delta)=h / \delta$. Therefore, from (8) and (9), we get $f / \delta=h_{k} / \delta$, which implies $f=h_{k} \in H$ as desired.

Corollary 3. $\mathscr{V}$ satisfies the $(3,2)$-transfer principle; i.e., there does not exist a three element interval $\theta_{0} \prec \theta_{1} \prec \theta_{2}$ with $\left\langle\operatorname{typ}\left(\theta_{0}, \theta_{1}\right), \operatorname{typ}\left(\theta_{1}, \theta_{2}\right)\right\rangle=\langle 3,2\rangle$ in any finite member $\mathbf{A}$ of $\mathscr{V}$.

Proof. Suppose, on the contrary, that such a three element interval $\theta_{0} \prec \theta_{1} \prec \theta_{2}$ exists in some $\mathbf{A} \in \mathscr{V}_{\text {fin }}$. We will get a contradiction. We work on Con $\mathbf{A}$. Let $\alpha_{0}$ be a maximal member of the set $\left\{\theta \in \operatorname{Con} \mathbf{A} \mid \theta \geq \theta_{0}, \theta \geq \theta_{1}\right\}$. Then $\alpha_{0}$ is strictly meet irreducible.

Let $\alpha_{1}=\theta_{1} \vee \alpha_{0}$ and $\alpha_{2}=\theta_{2} \vee \alpha_{0}$. Then $\theta_{2} \wedge \alpha_{0}=\theta_{0}$ since $I\left[\theta_{0}, \theta_{2}\right]$ is a three-element interval. We have just shown that $\left\langle\theta_{0}, \theta_{2}\right\rangle \nearrow\left\langle\alpha_{0}, \alpha_{2}\right\rangle$.

Now, the quotient algebra $\mathbf{A} / \alpha_{0} \in \mathscr{V}$ is subdirectly irreducible with monolith $\alpha_{1} / \alpha_{0}$. Moreover, by the tame congruence theory, the types of the quotients $\left\langle\alpha_{0} / \alpha_{0}, \alpha_{1} / \alpha_{0}\right\rangle$ and $\left\langle\alpha_{1} / \alpha_{0}, \alpha_{2} / \alpha_{0}\right\rangle$ are 3 and 2 respectively, which contradicts Theorem 2.

While we proved Corollary 3 and the following Theorem 4 for modular varieties, working independently and at about the same time, Valeriote [20] proved the $(3,2)$ and $(3,1)$-transfer principle for every finitely decidable locally finite variety. We incorporate Valeriote's result into our proof of the present Theorem 4 below.

Theorem 4. Let $\mathbf{A}$ be a finite subdirectly irreducible member of any finitely decidable variety with a monolith of type $\mathbf{3}$. Then $\mathrm{Con} \mathbf{A}$ is a linear lattice.

Proof. Suppose that Con $\mathbf{A}$ is not linear. In order to derive a contradiction, choose two incomparable congruences $\phi_{0}$ and $\phi_{1}$ of $\mathbf{A}$ so that $\phi_{0} \succ \phi_{0} \wedge \phi_{1} \stackrel{\text { def }}{=}$ $\phi \prec \phi_{1}$. Then $\operatorname{typ}\left(\phi, \phi_{i}\right)=3(i<2)$ by [20]. Let

$$
\mathbf{B} \stackrel{\text { def }}{=}\left\langle B, \leq, B_{0}, B_{1}\right\rangle
$$

be a finite Boolean triple. We are going to interpret $\mathbf{B}$ into some member of $P_{S, \text { fin }}(\mathbf{A})$ via a first order formula in a way which is independent of $\mathbf{B}$. That will suffice.

Wlog $B$ is the power set of some finite set $X$ and $\leq$ is simply the set theoretic inclusion relation. We define $\left\langle\mathbf{A}, \phi_{0}, \phi_{1}\right\rangle^{\mathbf{B}} \stackrel{\text { def }}{=} \mathbf{D}$ to be the subalgebra of $\mathbf{A}^{X}$ with the universe

$$
\left\{f \in A^{X} \mid f^{-1}\left(a / \phi_{i}\right) \in B_{i} \text { for every } a \in A, i<2\right\} .
$$

Indeed we can easily check that $D$ is a subuniverse from the fact that $A$ is finite. Further, we easily see that 
(10) For each $a \in A$, the constant map $\hat{a}$ belongs to $D$,

(11) $D$ is $\phi$-closed,

(12) If $\langle c, d\rangle \in \phi_{i}-\phi_{1-i}$, then

$$
\left.\left.\hat{c}\right|_{Y} \cup \hat{d}\right|_{X-Y} \in D \Leftrightarrow Y \in B_{1-i} \quad \forall i<2 .
$$

(Above construction is due to Idziak [9].) We recover $\mathbf{B}$ from $\mathbf{D}$ by the following scheme.

Let $\mu$ be the monolith of $\mathbf{A}$. Let $U$ be a $\left\langle 0_{A}, \mu\right\rangle$-minimal set with (the unique) trace $V \stackrel{\text { def }}{=}\{0,1\}$. Choose $e(x) \in \operatorname{Pol}_{1} \mathbf{A}$ so that $e \circ e=e$ and $e(A)=$ $U$, and choose $q(x, y) \in \mathbf{P o l}_{2} \mathbf{A}$ so that $\left.q\right|_{U}$ is the pseudo-join operation on $\left.\mathbf{A}\right|_{U}$. (see [7, (4.17)].) Then, for $a \in U$, we have $q(a, 1)=1 \Leftrightarrow a \in V$. Thus $D(V)$ is definable in $\mathbf{D}$ by the formula $e(x) \approx x \wedge q(\hat{1}, x) \approx \hat{1}$. Let $\Phi: D(V) \rightarrow B$ be the map given by $\Phi(f)=f^{-1}(1)$. Then $\Phi$ is onto by (10) and (11). It is obvious that $\Phi$ is one-to-one. Moreover, for $f, g \in D(V)$, we have

$$
\Phi(f) \subseteq \Phi(g) \Leftrightarrow q(f, g)=g .
$$

This $\langle B, \leq\rangle$ has been recovered.

In order to recover $B_{i} \quad(i<2)$, let $U_{i}$ be a $\left\langle\phi, \phi_{i}\right\rangle$-minimal set with (the unique) trace $V_{i} \stackrel{\text { def }}{=}\left\{0_{i}, 1_{i}\right\}$. Then, as we did for $\left\langle 0_{A}, \mu\right\rangle$, each $D\left(V_{i}\right)$ is definable in $\mathbf{D}$ by a first order formula. The following argument is for each $i<2$. Note that, since $\left\langle 0_{i}, 1_{i}\right\rangle \in \phi_{i}-\phi_{1-i}$, we have, for all $f \in V_{i}^{X}$,

$$
f \in D\left(V_{i}\right) \Leftrightarrow f^{-1}\left(1_{i}\right) \in B_{1-i}
$$

by (12). Since $\langle 0,1\rangle \in \mathrm{Cg}^{\mathbf{A}}\left(0_{i}, 1_{i}\right)$, using a usual Maltsev chain argument together with the trick of composing the idempotent unary polynomial $e(x)$, we can get a $\lambda_{i}(x) \in \mathrm{Pol}_{1} \mathbf{A}$ such that $\left\langle\lambda_{i}\left(0_{i}\right), \lambda_{i}\left(1_{i}\right)\right\rangle=\left\langle 0,1^{\prime}\right\rangle$ for some $1^{\prime} \in$ $U-\{0\}$. We claim that, for all $f \in D(V)$.

$$
\begin{aligned}
& f^{-1}(1) \in B_{i} \\
& \quad \Leftrightarrow\left(\exists g \in D\left(V_{1-i}\right)\right)(\forall h \in D(V))\left(q\left(h, \lambda_{1-i}(g)\right)=\lambda_{1-i}(g) \Leftrightarrow g(h, f)=f\right) .
\end{aligned}
$$

To prove the claim, suppose that $f \in D(V)$ and $f^{-1}(1)=\Phi(f) \in B_{i}$. Choose $g \in D\left(V_{1-i}\right)$ so that $g^{-1}\left(1_{1-i}\right)=f^{-1}(1)$. Then it is straightforward to check that this $g$ is a witness for the r.h.s. of (13). The converse of the claim is also easily proved. Thus we can recover the full structure $\left\langle B, \leq, B_{0}, B_{1}\right\rangle$ from $\mathbf{D}$. This completes the proof.

Proposition 5. Let $\mathscr{W}$ be a locally finite congruence modular variety, and let

$$
S_{3}=\left\{\mathbf{A} \in \mathscr{W}_{\text {fin }} \mid \mathbf{A} \text { is s.i. with monolith of type } 3\right\} \text {. }
$$

Then

$$
\mathscr{W}_{\text {fin }} \text { decidable } \nRightarrow \mathrm{V}\left(S_{3}\right) \text { congruence distributive. }
$$

In fact, we cannot have a decomposition theorem $\mathscr{W}=\mathscr{W}_{0} \otimes \mathscr{W}_{1}$ with $\mathscr{W}_{0}$ nontrivial and equal to either $\{\mathbf{A} \in \mathscr{W} \mid \mathbf{A}$ is abelian $\}$ or $\{\mathbf{A} \in \mathscr{W} \mid \mathbf{A}$ is congruence distributive\}.

Proof. We will construct a finite algebra $\mathbf{A}$ such that

(i) $\mathrm{V}(\mathbf{A})$ is finitely decidable and congruence permutable,

(ii) $\mathbf{A}$ is simple and $\operatorname{typ}\left(0_{A}, 1_{A}\right)=3$,

(iii) A has a nontrivial abelian subalgebra $\mathbf{B}$. 
Then $\mathscr{W} \stackrel{\text { def }}{=} \mathrm{V}(\mathbf{A})$ will be a witness of our proposition, i.e., $\mathscr{W}$ is locally finite, congruence modular and finitely decidable but $\mathrm{V}\left(S_{3}\right)$ is not congruence distributive and contains a nontrivial abelian algebra $\mathbf{B}$.

Let $A=\{0,1,2\}$ and $B=\{0,1\} \subseteq A$. The language of $\mathbf{A}$ has exactly one function symbol $\mathbf{d}$, which is ternary. The basic operation $d(x, y, z)=\mathbf{d}^{\mathbf{A}}$ is defined to be a Maltsev operation on $A$ such that

$$
\begin{array}{llll}
d(0,1,0)=1, & d(0,1,2)=1, & d(0,2,0)=1, & d(0,2,1)=1, \\
d(1,0,1)=0, & d(1,0,2)=0, & d(1,2,0)=2, & d(1,2,1)=0, \\
d(2,0,1)=1, & d(2,0,2)=0, & d(2,1,0)=0, & d(2,1,2)=2 .
\end{array}
$$

Then $\mathscr{W}$ is congruence permutable and hence modular. It is clear that $\mathbf{B}$ is a nontrivial subalgebra of $\mathbf{A}$. Actually, $\mathbf{B}$ is the only nontrivial subalgebra of $\mathbf{A}$ since $d(0,2,0)=1$ and $d(1,2,1)=0$. To show that $\mathbf{B}$ is abelian, it suffices to find an abelian algebra $\mathbf{B}^{\prime}$ with the same universe $B$ and Clo $^{\prime} \supseteq$ Clo $\mathbf{B}$. To this end, let $\mathbf{B}^{\prime}=\langle B,+\rangle$ be the usual two-element group. Then observe that $d(x, y, z)=x+y+z$ for every $z, y, z \in B$. (In fact, we have $\operatorname{Clo}^{\prime}=$ Clo B.)

We see that $\mathrm{Cg}^{\mathbf{A}}(0,1)=1_{A}$ from $d(0,2,0)=1$ and $d(1,2,0)=2$. Also $\mathrm{Cg}^{\mathbf{A}}(1,2)=1_{A}$ since $d(1,2,0)=2$ and $d(2,2,0)=0$, and $\mathrm{Cg}^{\mathbf{A}}(0,2)=1_{A}$ since $d(0,2,0)=1$ and $d(2,0,2)=0$. We have shown that $\mathbf{A}$ is simple.

A is not abelian since $d(1,2,0)=d(2,2,2)$ but $d(1,0,0) \neq d(2,0,2)$ which shows that $\langle 0,2\rangle$ is not in the center of $\mathbf{A}$. So $\operatorname{typ}\left(0_{A}, 1_{A}\right) \in\{\mathbf{3}, \mathbf{4}, \mathbf{5}\}$. But the presence of the Maltsev term operation $d$ excludes the possibility of $\operatorname{typ}\left(0_{A}, 1_{A}\right)$ being equal to one of 4 or 5 .

It remains to show that $\mathscr{W}$ is finitely decidable. For that matter, it suffices to show that $\mathscr{V}$ is directly representable. But this is immediate by (5.11) of [15].

\section{Abelian monolith CASE}

We return to considering a fixed congruence modular and finitely decidable variety $\mathscr{V}$.

Lemma 6. Let $\mathbf{A}$ be a finite subdirectly irreducible member of $\mathscr{V}$ with an abelian monolith $\mu$. If $\nu$ and $\theta$ are congruences of $\mathbf{A}$ such that $\theta \leq \nu=\left(0_{A}: \theta\right)$ and $\theta=\left(0_{A}: \nu\right)$, then the interval sublattice $\mathrm{I}\left[\nu, 1_{A}\right]$ is linear and $\operatorname{typ}\left\{\nu, 1_{A}\right\} \subseteq$ $\{3\}$.

Proof. Let $\mathbf{A} \in \mathscr{V}_{\mathrm{SI}}$, fin and $\mu, \nu, \theta \in \mathrm{Con} \mathbf{A}$ be given as in the hypotheses of the theorem. Let $\operatorname{Tht}(x, y)$ be a first order formula such that, whenever $\mathbf{D} \leq_{\text {diag }} \mathbf{A}^{\mathbf{x}}, f, g \in D$,

$$
\mathbf{D} \vDash \operatorname{Tht}(f, g) \Leftrightarrow\langle f, g\rangle \in D(\theta) .
$$

Such a formula $\operatorname{Tht}(x, y)$ exists by the remark following (7). We define two more formulas as follows.

$$
\begin{aligned}
& \operatorname{Img}(x, y, z, w)=\bigvee_{\lambda \in \mathrm{Pol}_{1} \mathbf{A}}(\lambda(z) \approx x \wedge \lambda(w) \approx y), \\
& \begin{aligned}
\operatorname{Red}(x, y, z, w)= & \bigvee_{s \in \mathrm{Pol}_{2} \mathbf{A}}(\exists u)(\exists v)(\operatorname{Tht}(u, v) \wedge s(z, u) \approx x \wedge s(z, v) \\
& \approx y \wedge s(w, u) \approx s(w, v)) .
\end{aligned}
\end{aligned}
$$


Then, for each $a, b \in A$, let

$$
\operatorname{Dom}_{a, b}(x, y)=\bigvee_{c \in A}(\exists w)(\operatorname{Img}(\hat{a}, x, \hat{c}, w) \wedge \operatorname{Red}(\hat{c}, w, \hat{b}, y)) \text {. }
$$

Claim 1. If $\mathbf{D} \leq_{\operatorname{diag}} \mathbf{A}^{X}$ is $\theta$-closed, then for all $f, g \in D, a, b \in A$,

(i) $\mathbf{D} \vDash \operatorname{Dom}_{a, b}(f, g) \Rightarrow \llbracket f \neq \hat{a} \rrbracket \subseteq \llbracket\langle g, \hat{b}\rangle \notin \nu \rrbracket$.

(ii) If $x \in X, \llbracket f \neq \hat{a} \rrbracket=\{x\}, f(x) \in a / \mu$ and $g(x) \notin b / \nu$, then $\mathbf{D} \vDash$ $\operatorname{Dom}_{a, b}(f, g)$.

Proof of Claim. 1. To prove (i), assume that $f, g \in D, \mathbf{D} \vDash \operatorname{Dom}_{a, b}(f, g)$, and suppose that $x \in X$ and $g(x) \stackrel{\nu}{=} b$. We want to show that $f(x)=a$. From the assumption $\mathbf{D} \vDash \operatorname{Dom}_{a, b}(f, g)$, we can find $c \in A$ and $h \in D$ such that

$$
\mathbf{D} \vDash \operatorname{Img}(\hat{a}, f, \hat{c}, h) \wedge \operatorname{Red}(\hat{c}, h, \hat{b}, g) .
$$

It suffices to show that $h(x)=c$. From $\mathbf{D} \vDash \operatorname{Red}(\hat{c}, h, \hat{b}, g)$, we can find $s \in \mathrm{Pol}_{2} \mathbf{A}$ and $\left\langle k_{u}, k_{v}\right\rangle \in D(\theta)$ such that

$$
\begin{gathered}
s\left(b, k_{u}(x)\right)=c, \quad s\left(b, k_{v}(x)\right)=h(x), \\
s\left(g(x), k_{u}(x)\right)=s\left(g(x), k_{v}(x)\right) .
\end{gathered}
$$

Then, since $\langle b, g(x)\rangle \in \nu$ and $\nu$ centralizes $\theta$, we must have

$$
h(x)=s\left(b, k_{v}(x)\right)=s\left(b, k_{u}(x)\right)=c,
$$

which completes the proof of (i).

Next, in order to prove (ii), we will assume the hypotheses and find $c \in$ $A, h \in D$ such that $\mathbf{D} \vDash \operatorname{Img}(\hat{a}, f, \hat{c}, h) \wedge \operatorname{Red}(\hat{c}, h, \hat{b}, g)$. Note that, for $\operatorname{Img}(\hat{a}, f, \hat{c}, h)$, it suffices to have $\llbracket h \neq \hat{c} \rrbracket=\{x\}$ since $\langle f(x), a\rangle \in \mu \subseteq$ any nonzero congruence of $\mathbf{A}$. Now, use the hypothesis $g(x) \notin b / \nu$ and (6) to find $s \in \operatorname{Pol}_{2} \mathbf{A},\langle u, v\rangle \in \theta$ such that $s(g(x), u)=s(g(x), v)$ and $s(b, u) \neq s(b, v)$. Then, if we let $k \in A^{X}$ be given by

$$
k=\left.\left.\hat{u}\right|_{X-\{x\}} \cup \hat{v}\right|_{\{x\}},
$$

then $k \in D$ as $D$ is $\theta$-closed. Finally, let $c=s(b, u) \in A$ and let $h=s(\hat{b}, k) \in$ $D$. Then $\mathbf{D} \vDash \operatorname{Img}(\hat{a}, f, \hat{c}, h)$ since $\llbracket h \neq \hat{c} \rrbracket=\{x\}$, and $\mathbf{D} \vDash \operatorname{Red}(\hat{c}, h, \hat{b}, g)$ since

$$
\operatorname{Tht}(\hat{u}, k) \wedge s(\hat{b}, \hat{u}) \approx \hat{c} \wedge s(\hat{b}, k) \approx h \wedge s(g, \hat{u}) \approx s(g, k)
$$

holds in $\mathbf{D}$ by construction.

Claim 2. If $\alpha$ is a congruence of $\mathbf{A}$ such that $\nu \prec \alpha$, then $\operatorname{typ}(\nu, \alpha)=\mathbf{3}$.

Proof of Claim. 2. Suppose, on the contrary, that $\operatorname{typ}(\nu, \alpha)=2$. We will semantically embed the class of all finite graphs into $\mathrm{P}_{\mathrm{S}, \text { fin }}(\mathbf{A})$. Choose a $\langle\nu, \alpha\rangle$ trace $T$ and an ordered pair $\langle 0,1\rangle \in T^{2}-\nu$. Let $U$ be the $\langle\nu, \alpha\rangle$-minimal set containing $T$. Let a finite graph $\mathbf{G} \stackrel{\text { def }}{=}\langle V, E\rangle$ be given. Wlog $V \cap E=\varnothing$. Choose $x_{0} \neq x_{1}$ so that $x_{i} \notin V \cup E(i<2)$. Let $X=V \cup E \cup\left\{x_{0}, x_{1}\right\}$. For each $v \in V$, define $h_{v} \in A^{X}$ by

$$
\begin{aligned}
h_{v}(x) & =1 & & \text { if } x=v \text { or } \\
& =0 & & \text { otherwise. }
\end{aligned}
$$


Define one more map $h_{V} \in A^{X}$ by

$$
\begin{aligned}
h_{V}(x) & =1 & & \text { if } x \in V \text { or } x=x_{1}, \\
& =0 & & \text { otherwise. }
\end{aligned}
$$

Let

$$
\begin{aligned}
D^{*} & =\operatorname{Sg}^{\mathbf{A}^{X}}\left(\left\{h_{v} \mid v \in V\right\} \cap\left\{h_{V}\right\} \cup\{\hat{a} \mid a \in A\}\right), \\
D & =\left\{f \in A^{X} \mid\langle f, g\rangle \in D(\nu) \text { for some } g \in D^{*}\right\} .
\end{aligned}
$$

Then $\mathbf{D}$ is a $\nu$-closed diagonal subdirect power of $\mathbf{A}$.

Fix $\langle c, d\rangle \in \mu-0_{A}$ and let, for each $i<2$,

$$
k_{i}=\left.\left.\hat{c}\right|_{X-\left\{x_{i}\right\}} \cup \hat{d}\right|_{\left\{x_{i}\right\}} \text {. }
$$

Note that $k_{0}, k_{1} \in D$ as $D$ is $\nu$-closed.

Let

$$
\begin{aligned}
& \operatorname{Eq}(x, y)=(\exists u)(u \not \approx \hat{c} \wedge \operatorname{Img}(\hat{c}, u, \hat{c}, x) \wedge \operatorname{Img}(\hat{c}, u, \hat{c}, y)), \\
& \operatorname{Dom}^{\prime}(x, y)=x \not \approx \hat{c} \wedge \operatorname{Dom}_{c, 0}\left(x, h_{V}\right) \wedge \operatorname{Dom}_{c, 0}(x, y) .
\end{aligned}
$$

Using (1) and the fact that $\mu$ is below any nonzero congruence of $\mathbf{A}$, we can easily show that, if $f$ and $g$ are members of $D$ such that $|\mathbb{L} f \neq \hat{c} \rrbracket|=$ $|\llbracket g \neq \hat{c} \rrbracket|=1$, we have

$$
\mathbf{D} \vDash \operatorname{Eq}(f, g) \Leftrightarrow \llbracket f \neq \hat{c} \rrbracket=\llbracket g \neq \hat{c} \rrbracket .
$$

Let $\operatorname{Vor}(x)$ be a first order formula saying that

$$
\begin{aligned}
x \in D(U) \& \neg \operatorname{Dom}_{c, 0}\left(k_{0}, x\right) \& \neg \operatorname{Dom}_{c, 0}\left(k_{1}, x\right) \&(\exists y) \operatorname{Dom}^{\prime}(y, x) \\
\quad \&\left[(\forall y, z)\left(\operatorname{Dom}^{\prime}(y, x) \& \operatorname{Dom}^{\prime}(z, x) \Rightarrow \mathrm{Eq}(y, z)\right)\right],
\end{aligned}
$$

and let

$$
\text { Vor }=\{f \in D \mid \mathbf{D} \vDash \operatorname{Vor}(f)\} .
$$

Note that if $f \in D$, then $\langle f(x), f(y)\rangle \in \alpha$ for all $x, y \in X$ since each generator of $D$ behaves like this. Thus, if $f \in D(U)$, then we actually have $f \in$ $D\left(T^{\prime}\right)$ for some $\langle\nu, \alpha\rangle$-trace $T^{\prime} \subseteq U$. Suppose that $f \in D\left(T^{\prime}\right)$, where $T^{\prime} \subseteq U$ is some $\langle\nu, \alpha\rangle$-trace different from $T$. Then $f\left(x_{0}\right) \notin 0 / \nu$ and hence $\mathbf{D} \vDash$ $\operatorname{Dom}_{c, 0}\left(k_{0}, f\right)$ by Claim 1. Since $\neg \operatorname{Dom}_{c, 0}\left(k_{0}, x\right)$ is a conjunct of $\operatorname{Vor}(x)$, we see that $f \notin V$ or. We have shown $\operatorname{Vor} \subseteq D(T)$.

Let $V=\left\{v_{i} \mid i<m\right\}$ and let $h_{i}=h_{v_{i}}(i<m)$. Let $f \in V$ or be given. Then we can choose $\lambda \in \operatorname{Pol}_{1} \mathbf{A}$ so that $\left\langle f, \lambda\left(h_{0}, \ldots, h_{m-1}, h_{V}\right)\right\rangle \in D(\nu)$. Wlog $\lambda$ is a polynomial of the induced algebra $\left.\mathbf{A}\right|_{T}$. But $\left(\left.\mathbf{A}\right|_{T}\right) /\left(\left.\nu\right|_{T}\right)$ is polynomially equivalent to a module, say $\mathbf{M} \stackrel{\text { def }}{=}\left\langle T / \nu,+,-, 0^{\mathbf{M}}, r(r \in R)\right\rangle$. We may assume that $0 / \nu=0^{\mathbf{M}}$. Hence, for some ring elements $r_{i}(i \leq m)$ and for some $a \in T$, we have

$$
f / \nu=\sum_{i<m} r_{i} \cdot\left(\frac{h_{i}}{\nu}\right)+r_{m} \cdot\left(\frac{h_{V}}{\nu}\right)+\frac{a}{\nu} .
$$

By evaluating this identity at $x_{i}(i<2)$, we get $a / \nu=0^{\mathbf{M}}$ and $r_{m} \cdot(1 / \nu)=$ $0^{\mathbf{M}}$. Thus $f / \nu=\sum_{i<m} r_{i} \cdot\left(h_{i} / \nu\right)$. So we have, for each $j<m$ and for each $e \in E$,

$$
(f / \nu)\left(v_{j}\right)=r_{j} \cdot(1 / \nu)
$$




$$
(f / \nu)(e)=\sum\left\{r_{j} \cdot(1 / \nu) \mid v_{i} \in e\right\} .
$$

We assert that $r_{j} \cdot(1 / \nu)=0^{\mathrm{M}}$ for all but one $j<m$. We first see that $r_{j} \cdot(1 / \nu) \neq 0^{\mathbf{M}}$ for at least one $j<m$ from Claim 1(i) and $\mathbf{D} \vDash(\exists y) \operatorname{Dom}^{\prime}(y, f)$ (which is a part of $\mathbf{D} \vDash \operatorname{Vor}(f)$ ). To prove the uniqueness of such $j$, let us assume that $r_{j} \cdot(1 / \nu) \neq 0^{\mathbf{M}} \neq r_{j^{\prime}} \cdot(1 / \nu)$ for some $j<j^{\prime}<m$. Then $f\left(v_{j}\right) \notin 0 / \nu$ and $f\left(v_{j^{\prime}}\right) \notin 0 / \nu$ by (17). Thus, if we let $g_{p}=\left.\left.\hat{c}\right|_{X-\left\{v_{p}\right\}} \cup \hat{d}\right|_{\left\{v_{p}\right\}}$ $\left(p=j, j^{\prime}\right)$, then we have $\mathbf{D} \vDash \operatorname{Dom}^{\prime}\left(g_{j}, f\right) \wedge \operatorname{Dom}^{\prime}\left(g_{j^{\prime}}, f\right) \wedge \neg \mathrm{Eq}\left(g_{j}, g_{j^{\prime}}\right)$ which contradicts $\mathbf{D} \vDash \operatorname{Vor}(f)$. Thus the assertion is proved. Therefore, by (17) and (18), $f / \nu=r_{k} \cdot\left(h_{k} / \nu\right)$ for some $k<m, r_{k}$ such that $r_{k} \cdot(1 / \nu) \neq 0 / \nu$. Observe that

$$
\llbracket\langle f, \hat{0}\rangle \notin \nu \rrbracket=\llbracket\left\langle h_{k}, \hat{0}\right\rangle \notin \nu \rrbracket .
$$

Let $\Phi: V o r \rightarrow V$ be the map $f \mapsto v_{k}$, where $f$ and $k$ are related as above. We see this map $\Phi$ is onto since each $h_{i} \in V$ or and $\Phi\left(h_{i}\right)=v_{i}$. Let

$$
\operatorname{Eqv}(x, y)=(\exists u)\left(\operatorname{Dom}^{\prime}(u, x) \wedge \operatorname{Dom}^{\prime}(u, y)\right),
$$

$\operatorname{Edge}(x, y)=\neg \operatorname{Eqv}(x, y) \wedge(\exists u)\left(u \neq \hat{c} \wedge \operatorname{Dom}_{c, 0}(u, x) \wedge \operatorname{Dom}_{c, 0}(u, y)\right)$.

Then, it is straightforward to check that, for all $f, f^{\prime} \in V o r$,

$$
\begin{gathered}
\mathbf{D} \vDash \operatorname{Eqv}\left(f, f^{\prime}\right) \Leftrightarrow \Phi(f)=\Phi\left(f^{\prime}\right), \\
\mathbf{D} \vDash \operatorname{Edge}\left(f, f^{\prime}\right) \Leftrightarrow\left\{\Phi(f), \Phi\left(f^{\prime}\right)\right\} \in E .
\end{gathered}
$$

The semantic embedding is now completed.

Claim. 3. In Con $\mathbf{A}$, we cannot have two distinct covers $\alpha_{0}, \alpha_{1}$ of $\nu$.

Proof of Claim. 3. Suppose that $\alpha_{0} \neq \alpha_{1}$ and $\alpha_{0} \succ \nu, \alpha_{1} \succ \nu$. By Claim 2, we know that both of the prime quotients have type 3 . We will interpret the following class of relational structures into $\mathrm{P}_{\mathrm{S}, \mathrm{fn}}(\mathbf{A})$.

$$
\operatorname{ED}_{\text {fin }} \stackrel{\text { def }}{=}\left\{\left\langle X, \rho_{0}, \rho_{1}\right\rangle\left|\rho_{0}, \rho_{1} \in \operatorname{Eqv}(X), \rho_{0} \cap \rho_{1}=0_{X} \& 0<\right| X \mid<\omega\right\} .
$$

Let $\mathbf{X} \stackrel{\text { def }}{=}\left\langle X, \rho_{0}, \rho_{1}\right\rangle \in \mathrm{ED}_{\text {fin }}$ be given. Let $\mathbf{P}(X)$ be the power set Boolean algebra on the set $X$, and for each $i<2$ let $\mathbf{B}_{i}=$ the sub-Boolean algebra of $\mathbf{P}(X)$ generated by the $\rho_{i}$-classes. Let

$$
A(\mathbf{X})=\left\{f \in A^{X} \mid f^{-1}\left(a / \alpha_{i}\right) \in B_{i}(\forall a \in A, \forall i<2)\right\} .
$$

We are going to semantically embed $\mathbf{X}$ into $\mathbf{A}(\mathbf{X}) \leq_{\text {diag }} \mathbf{A}^{X}$.

In the following discussion, $i$ is a variable ranging over $\{0,1\}$. Choose a $\left\langle\nu, \alpha_{i}\right\rangle$-minimal set $\left\{0_{i}, 1_{i}\right\}$, a unary idempotent polynomial operation $e_{i}$ of $\mathbf{A}$ such that $e_{i}(A)=\left\{0_{i}, 1_{i}\right\}$ and a binary polynomial operation meet $_{i}$ of $\mathbf{A}$ such that meet $\left.\right|_{\left.i 0_{i}, 1_{i}\right\}}$ is the Boolean meet operation of $\mathbf{A}_{\left\{0_{i}, 1_{i}\right\}}$. Since $\left\langle 0_{i}, 1_{i}\right\rangle \in \alpha_{i}-\alpha_{1-i}$, we only have to find formulas $\Delta_{i}(x), \Gamma_{i}(x)$, and $\Gamma(x, y)$ such that for all $f, g \in D$,

$$
\begin{aligned}
& \mathbf{D} \vDash \Delta_{i}(f) \text { iff } f \in D\left(\left\{0_{i}, 1_{i}\right\}\right), \\
& \mathbf{D} \vDash \Gamma_{i}(f) \text { iff } f \in D\left(\left\{0_{i}, 1_{i}\right\}\right) \& f^{-1}\left(1_{i}\right) \text { is a } \rho_{1-i} \text {-block, } \\
& \mathbf{D} \vDash \Gamma(f, g) \text { iff } \mathbf{D} \vDash \Gamma_{0}(f) \wedge \Gamma_{1}(g) \& f^{-1}\left(1_{0}\right) \cap g^{-1}\left(1_{1}\right) \neq \varnothing .
\end{aligned}
$$


For the details of this interpretation scheme, see [10]. Let

$$
\begin{aligned}
\Delta_{i}(x) & =e_{i}(x) \approx x, \\
\Gamma_{i}(x) & =\Delta_{i}(x) \wedge(\forall y)\left(\left(\Delta_{1}(y) \wedge \operatorname{meet}_{i}(x, y) \approx y \wedge y \not \hat{0}_{i}\right) \rightarrow y \approx x\right), \\
\Gamma(x, y) & =\Gamma_{0}(x) \wedge \Gamma_{1}(y) \wedge(\exists u)\left(u \not c \wedge \operatorname{Dom}_{c}, 0_{0}(u, x) \wedge \operatorname{Dom}_{c, 0_{1}}(u, y)\right) .
\end{aligned}
$$

It is straightforward to check that these formulas do the job.

We have shown that $\mathbf{A} / \nu$ is either trivial or subdirectly irreducible with type 3 monolith. Thus, by Theorem 2 and Theorem 4, the proof of the lemma has been completed.

Remark. The statement of Lemma 6 Claim 2 was first conjectured by R. Willard. In fact, he proved the statement himself (assuming permutability) independent of the author [21].

The following lemma is an expanded version of (7.8) of [6].

Lemma 7. Let $\mathbf{A}$ be a finite subdirectly irreducible algebra in a congruence modular variety. Let $\mu$ be the monolith of $\mathbf{A}$ and suppose that $\left(0_{A}: \mu\right) \stackrel{\text { def }}{=} \nu>\theta \stackrel{\text { def }}{=}$ $\left(0_{A}: \nu\right)$. Then there exists a finite subdirectly irreducible algebra $\mathbf{A}^{*}$ with monolith $\mu^{*}$ in the variety $\mathrm{V}(\mathbf{A})$ such that

(i) $\left(0_{A}: \mu^{*}\right) \stackrel{\text { def }}{=} \nu^{*}>\theta^{*} \stackrel{\text { def }}{=}\left(0_{A}: \nu^{*}\right)$.

(ii) There exist $\alpha_{i}, \alpha_{i}^{\prime}(i<4), \beta, \beta^{\prime} \in$ Con $\mathbf{A}^{*}$ (all distinct) such that

(a) $\alpha_{0} \vee \alpha_{1}=\alpha_{2} \vee \alpha_{3}=\beta \vee \beta^{\prime}=\nu^{*}$,

(b) $\alpha_{0}^{\prime} \wedge \alpha_{1}^{\prime}=\alpha_{2}^{\prime} \wedge \alpha_{3}^{\prime}=\beta \wedge \beta^{\prime}=\theta^{*}$,

(c) $\alpha_{0} \wedge \alpha_{1}=\alpha_{2}^{\prime} \vee \alpha_{3}^{\prime}=\beta$,

(d) $\alpha_{2} \wedge \alpha_{3}=\alpha_{0}^{\prime} \vee \alpha_{1}^{\prime}=\beta^{\prime}$,

(e) $\alpha_{i}=\left(0_{A^{*}}: \alpha_{i}^{\prime}\right)(i<4), \alpha_{i}^{\prime}=\left(0_{A^{*}}: \alpha_{i}\right)(i<4)$,

(f) $\beta=\left(0_{A^{*}}: \beta^{\prime}\right), \beta^{\prime}=\left(0_{A^{*}}: \beta\right)$,

(g) if $\langle\theta, \nu\rangle$ is not solvable, then none of the following quotients are solvable:

$$
\begin{array}{llll}
\left\langle\alpha_{i}, \nu^{*}\right\rangle & (i<4), \quad\left\langle\beta, \alpha_{i}\right\rangle,\left\langle\alpha_{i}^{\prime}, \beta^{\prime}\right\rangle \quad(i=0,1), \\
\left\langle\beta^{\prime}, \alpha_{i}\right\rangle, & \left\langle\alpha_{i}^{\prime}, \beta\right\rangle \quad(i=2,3), \quad\left\langle\theta^{*}, \alpha_{i}\right\rangle \quad(i<4) .
\end{array}
$$

Figure 1 shows a part of $\operatorname{Con} \mathbf{A}^{*}$.

Proof. We adopt the following notation: If $\mathbf{B}$ is an algebra and $\mathbf{C}$ is a subdirect subalgebra of $\mathbf{B}^{2}$, then for each congruence $\delta$ of $\mathbf{B}$, and for each $i<2$, $\delta_{i}$ is the preimage of $\delta$ under the projection map $\pi_{i}: C \rightarrow B$ onto the $i$ th coordinate. If $\delta, \theta \in$ Con $\mathbf{B}$, then $\delta \times \theta$ is defined to be the congruence $\delta_{0} \wedge \theta_{1}$ of $\mathbf{C}$. For each $i<2, \eta_{i}$ is the kernel of $\pi_{i}$. We will use the symbols "." and "+" for the "meet" and "join" lattice operations respectively.

Let $\mathbf{A}$ and $\mu, \nu, \theta \in$ Con $\mathbf{A}$ be given as in the hypothesis. Note that $\nu>\theta \geq \mu \succ 0_{A}$. We are going to investigate the congruence lattice of the algebra $\mathbf{A}(\nu) \stackrel{\text { def }}{=} \mathbf{A}^{\prime}$. (The universe $A^{\prime}$ of the algebra $\mathbf{A}^{\prime}$ is $\nu \subseteq A^{2}$.) Let $\nu^{\prime}=\nu_{0} \cdot \nu_{1} \quad\left(=\nu_{0}=\nu_{1}\right)$, and let $\theta^{\prime}=\theta_{0} \cdot \theta_{1}$. Define

$$
\Delta=\mathrm{Cg}^{\mathbf{A}^{\prime}}(\{\langle\langle x, x\rangle,\langle y, y\rangle\rangle \mid\langle x, y\rangle \in \theta\}),
$$

and let $\mu^{\prime}=\Delta+\mu_{0} \cdot \mu_{1}$. (Figure 2 shows a part of Con $\mathbf{A}^{\prime}$.) Clearly, we have $\theta^{\prime} \geq \Delta$ and $\Delta+\eta_{i}=\theta_{i} \quad(i<2)$. Also it is easily verified that $\Delta \cdot \eta_{i}=0_{A^{\prime}}$ 


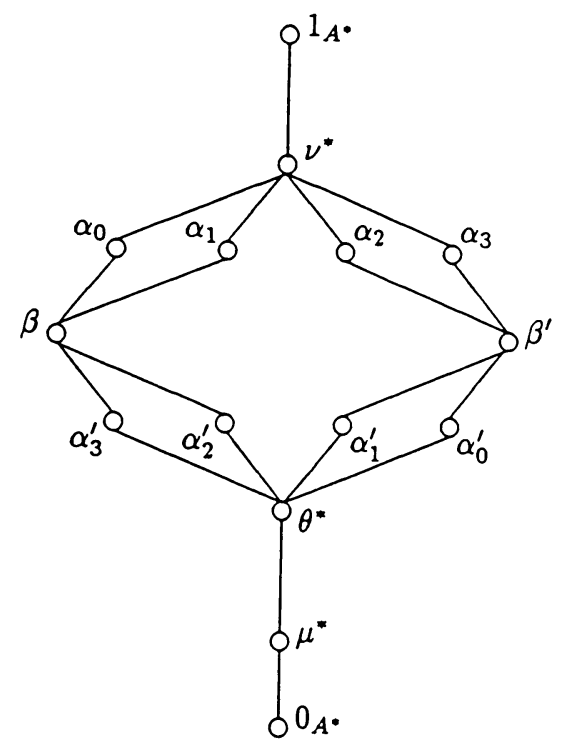

FIGURE 1

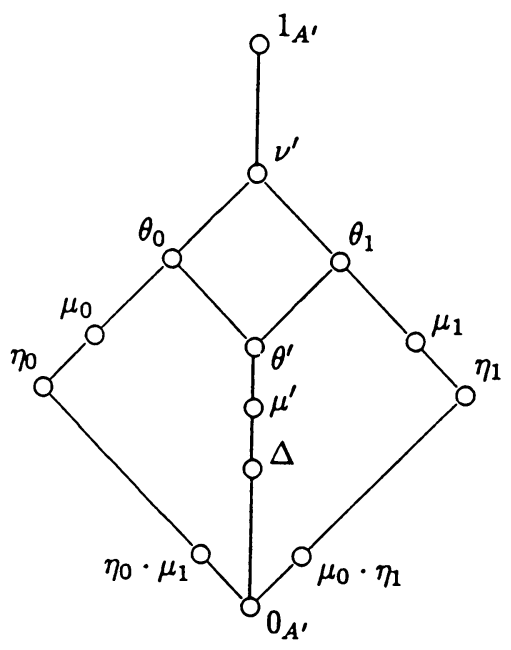

FIGURE 2

$(i<2)$ since the implications $\langle\langle x, u\rangle,\langle x, v\rangle\rangle \in \Delta \Rightarrow\langle u, v\rangle \in[\theta, \nu]=0_{A}$ (and similarly $\langle\langle u, x\rangle,\langle v, x\rangle\rangle \in \Delta \Rightarrow\langle u, v\rangle \in[\theta, \nu]$ ) holds by Theorem (4.9) of Freese and McKenzie [6]. Note that $\mu_{1}$ is the unique cover of $\eta_{1}$. But the interval $\mathrm{I}\left[\eta_{1}, \nu^{\prime}\right]$ projects down to $\mathrm{I}\left[0_{A^{\prime}}, \eta_{0}\right]$ which in turn projects up to $\mathrm{I}\left[\Delta, \theta_{0}\right]$. By the projectivity maps involved here, $\mu_{1}$ is mapped to $\Delta+\eta_{0} \cdot \mu_{1}$. Thus $\Delta+\eta_{0} \cdot \mu_{1}$ is the unique cover of $\Delta$ in $\mathrm{I}\left[\Delta, \theta_{0}\right]$. Similarly, $\Delta+\eta_{1} \cdot \mu_{0}$ is the unique cover of $\Delta$ of $\mathrm{I}\left[\Delta, \theta_{1}\right]$. Since $\Delta \leq \theta^{\prime}$ and $\mu_{i} \leq \theta_{i} \quad(i<2)$, we have $\Delta+\eta_{i} \cdot \mu_{1-i} \in I\left[\Delta, \theta_{1-i}\right] \quad(i<2)$. Thus $\Delta+\eta_{0} \cdot \mu_{1}=\Delta+\eta_{1} \cdot \mu_{0} \succ \Delta$. Since $\mu_{0} \cdot \mu_{1}=\eta_{0} \cdot \mu_{1}+\eta_{1} \cdot \mu_{0}$, we now have $\mu^{\prime}=\Delta+\eta_{0} \cdot \mu_{1}=\Delta+\eta_{1} \cdot \mu_{0} \succ \Delta$.

Claim. (i) $\mu^{\prime}$ is the unique cover of $\Delta$.

(ii) $\theta^{\prime}=\left(0_{A^{\prime}}: \nu^{\prime}\right), \theta^{\prime} / \Delta=\left(0_{A^{\prime} / \Delta}: \nu^{\prime} / \Delta\right)$.

(iii) $\nu^{\prime}=\left(0_{A^{\prime}}: \theta^{\prime}\right), \nu^{\prime} / \Delta=\left(0_{A^{\prime} / \Delta}: \theta^{\prime} / \Delta\right)=\left(0_{A^{\prime} / \Delta}: \mu^{\prime} / \Delta\right)$.

(iv) $\theta_{i}=\left(0_{A^{\prime}}: \theta_{1-i}\right), \theta_{i} / \Delta=\left(0_{A^{\prime} / \Delta}: \theta_{1-i} / \Delta\right) \quad(i<2)$,

(v) $\mathbf{I}\left[\theta_{i}, \nu^{\prime}\right] \cong \mathbf{I}\left[\theta^{\prime}, \theta_{i}\right] \cong \mathbf{I}[\theta, \nu]$ for each $i<2$. Moreover, if $\langle\theta, \nu\rangle$ is not solvable, then neither $\left\langle\theta_{i}, \nu^{\prime}\right\rangle$ nor $\left\langle\theta^{\prime}, \theta_{i}\right\rangle$ is solvable for each $i<2$.

To prove (i), suppose $\Delta<\gamma \in \operatorname{Con} \mathbf{A}^{\prime}$. It suffices to show that $\gamma \geq \eta_{0} \cdot \mu_{1}$ or $\gamma \geq \eta_{1} \cdot \mu_{0}$. Assume not. We will get a contradiction. Note that, by the argument preceding the Claim, it is enough to show that $\gamma \leq \theta_{0}$. From $\gamma \geq \eta_{0} \cdot \mu_{1}$, we get $\gamma \cdot \eta_{0}=0_{A^{\prime}}$ since $\eta_{0} \cdot \mu_{1}$ is the unique cover of $0_{A^{\prime}}$ below $\eta_{0}$. Similarly, we get $\gamma \cdot \eta_{1}=0_{A^{\prime}}$. Thus

$$
\left[\gamma, \nu^{\prime}\right]=\left[\gamma, \eta_{0}+\eta_{1}\right]=\left[\gamma, \eta_{0}\right]+\left[\gamma, \eta_{1}\right] \leq \gamma \cdot \eta_{0}+\gamma \cdot \eta_{1}=0_{A^{\prime}} .
$$

Now apply the commutator homomorphism theorem to the projection map $\pi_{0}: A^{\prime} \rightarrow A$ and the equality $\left[\gamma, \nu^{\prime}\right]=0_{A^{\prime}}$ to get

$$
\left[\pi_{0}\left(\gamma+\eta_{0}\right), \pi_{0}\left(\nu^{\prime}+\eta_{0}\right)\right]=\pi\left(\left[\gamma, \nu^{\prime}\right]+\eta_{0}\right)=\pi_{0}\left(\eta_{0}\right)=0_{A} .
$$

Thus $\left[\pi_{0}\left(\gamma+\eta_{0}\right), \nu\right]=0_{A}$. Hence $\pi_{0}\left(\gamma+\eta_{0}\right) \leq \theta$, which implies $\gamma \leq \theta_{0}$ as desired. This completes the proof of (i).

Observe that the above argument shows that for all $\gamma \in \operatorname{Con} \mathbf{A}^{\prime}$, if $\left[\gamma, \nu^{\prime}\right]=$ $0_{A^{\prime}}$, then $\gamma \leq \theta_{0} \cdot \theta_{1}=\theta^{\prime}$. This observation together with the fact that $\left[\theta^{\prime}, \nu^{\prime}\right]=$ 
$[\theta \times \theta, \nu \times \nu] \leq[\theta, \nu] \times[\theta, \nu]=0_{A^{\prime}}$ prove $\theta^{\prime}=\left(0_{A^{\prime}}: \nu^{\prime}\right)$. To prove the rest of (ii), $\theta^{\prime} / \Delta=\left(0_{A^{\prime} / \Delta}: \nu^{\prime} / \Delta\right)$, we only need to show $\left[\gamma, \nu^{\prime}\right] \leq \Delta \Rightarrow\left[\gamma, \nu^{\prime}\right]=0_{A^{\prime}}$, $\forall \gamma \in$ Con $\mathbf{A}^{\prime}$. So suppose $\left[\gamma, \nu^{\prime}\right]=\left[\gamma, \eta_{0}+\eta_{1}\right] \leq \Delta$. Then

$\left[\gamma, \eta_{0}+\eta_{1}\right] \leq \eta_{0}+\left[\gamma, \eta_{1}\right] \leq \eta_{0}+\left[\gamma, \nu^{\prime}\right] \cdot \eta_{1} \leq \eta_{0}+\Delta \cdot \eta_{1}=\eta_{0}+0_{A^{\prime}}=\eta_{0}$.

Similarly, we get $\left[\gamma, \eta_{0}+\eta_{1}\right] \leq \eta_{1}$. Hence $\left[\gamma, \nu^{\prime}\right]=\left[\gamma, \eta_{0}+\eta_{1}\right] \leq \eta_{0} \cdot \eta_{1}=$ $0_{A^{\prime}}$.This completes the proof of (ii).

To prove (iii), we only need to show $\left[\gamma, \mu^{\prime}\right] \leq \Delta \Rightarrow \gamma \leq \nu^{\prime}, \forall \gamma \in$ Con $\mathbf{A}^{\prime}$. Suppose that $\nu^{\prime}<\gamma \in$ Con $\mathbf{A}^{\prime}$. We will show that $\gamma$ cannot centralize $\mu^{\prime}$ modulo $\Delta$. That will suffice. Choose $\left\langle\langle a, b\rangle,\left\langle a^{\prime}, b^{\prime}\right\rangle\right\rangle \in \gamma$ so that $\left\langle a, a^{\prime}\right\rangle \notin \nu$. Then $\left\langle\langle a, a\rangle,\left\langle a^{\prime}, a^{\prime}\right\rangle\right\rangle \in \gamma$ since $\gamma \geq \eta_{0}$. Choose a term $t\left(x, y_{0}, \ldots, y_{n-1}\right)$ and $\left\langle c_{i}, d_{i}\right\rangle \in \mu \quad(i<n)$ so that $t(a, \bar{c})=t(a, \bar{d})$ and $t\left(a^{\prime}, \bar{c}\right) \neq t\left(a^{\prime}, \bar{d}\right)$. Now we have, $\left\langle\langle a, a\rangle,\left\langle a^{\prime}, a^{\prime}\right\rangle\right\rangle \in \gamma,\left\langle\left\langle c_{i}, c_{i}\right\rangle,\left\langle c_{i}, d_{i}\right\rangle\right\rangle \in \mu^{\prime} \quad(i<n)$ and

$$
\begin{gathered}
\left\langle t\left(\langle a, a\rangle,\left\langle c_{0}, c_{0}\right\rangle, \ldots,\left\langle c_{n-1}, c_{n-1}\right\rangle\right),\right. \\
\left.t\left(\langle a, a\rangle,\left\langle c_{0}, d_{0}\right\rangle, \ldots,\left\langle c_{n-1}, d_{n-1}\right\rangle\right)\right\rangle \in \Delta, \\
\left\langle t\left(\left\langle a^{\prime}, a^{\prime}\right\rangle,\left\langle c_{0}, c_{0}\right\rangle, \ldots,\left\langle c_{n-1}, c_{n-1}\right\rangle\right),\right. \\
\left.t\left(\left\langle a^{\prime}, a^{\prime}\right\rangle,\left\langle c_{0}, d_{0}\right\rangle, \ldots,\left\langle c_{n-1}, d_{n-1}\right\rangle\right)\right\rangle \in \eta_{0}-\eta_{1} .
\end{gathered}
$$

But the last membership relation " $\in \eta_{0}-\eta_{1}$ " implies " $\notin \Delta$ " since $\Delta \cdot \eta_{0}=$ $0_{A^{\prime}} \leq \eta_{1}$. We completed the proof of (iii).

To prove (iv), first observe that

$$
\begin{aligned}
{\left[\theta_{0}, \theta_{1}\right] } & =\left[\eta_{0}+\Delta, \eta_{1}+\Delta\right] \leq \eta_{0} \cdot \eta_{1}+\Delta \cdot \eta_{1}+\eta_{0} \cdot \Delta+[\Delta, \Delta] \\
& \leq[\Delta, \Delta] \leq\left[\nu^{\prime}, \theta^{\prime}\right]=0_{A^{\prime}} .
\end{aligned}
$$

Suppose that $\gamma \in$ Con $\mathbf{A}^{\prime}$ and $\left[\gamma, \theta_{1}\right] \leq \Delta$. Then $\left[\gamma, \eta_{1}\right] \leq\left[\gamma, \theta_{1}\right] \cdot \eta_{1} \leq$ $\Delta \cdot \eta_{1}=0_{A^{\prime}}$. By the homomorphism property, we get $\left[\pi_{0}\left(\gamma+\eta_{0}\right), \nu\right]=0_{A}$. Hence $\gamma \leq \theta_{0}$. We have shown $\theta_{0}=\left(0_{A^{\prime}}: \theta_{1}\right)$ and $\theta_{0} / \Delta=\left(0_{A^{\prime} / \Delta}: \theta_{1} / \Delta\right)$. By a symmetric argument, we are done with (iv).

To prove (v), just observe that, for each $i<2$, both the projection map $\pi_{i}: \mathbf{I}\left[\theta_{i}, \nu^{\prime}\right] \rightarrow \mathbf{I}[\theta, \nu]$ and the projectivity map $\mathbf{I}\left[\theta^{\prime}, \theta_{1-i}\right] \rightarrow \mathbf{I}\left[\theta_{i}, \nu^{\prime}\right]$ are lattice isomorphisms that preserve solvability of congruence quotients.

Now we have a finite subdirectly irreducible algebra $\mathbf{A}^{\prime} / \Delta \in \mathrm{V}(\mathbf{A})$ with monolith $\mu^{\prime} / \Delta$ and $\left(0_{A^{\prime}}: \mu^{\prime} / \Delta\right)=\nu^{\prime} / \Delta>\theta^{\prime} / \Delta=\left(0_{A^{\prime} / \Delta}: \nu^{\prime} / \Delta\right)$. So we can apply the result of the Claim to $\mathbf{A} / \Delta$ (in place of $\mathbf{A}$ ). Let us rename $\mathbf{A}^{\prime} / \Delta, \mu / \Delta, \nu^{\prime} / \Delta$, $\theta_{0} / \Delta$, and $\theta_{1} / \Delta$ as $\mathbf{A}, \mu, \nu, \theta, \phi$, and $\psi$ respectively. We are going to investigate the congruence lattice of the algebra $\mathbf{A}(\nu) \stackrel{\text { def }}{=} \mathbf{A}^{\prime}$. Let $\nu^{\prime}, \theta^{\prime}, \mu^{\prime}, \Delta$ be defined as before. This time, we have additional congruences $\phi_{0}, \phi_{1}, \psi_{0}, \psi_{1}$. Finally, let

$$
\begin{aligned}
\mathbf{A}^{*} & =\mathbf{A}^{\prime} / \Delta, \\
\nu^{*} & =\nu^{\prime} / \Delta, \quad \theta^{*}=\theta^{\prime} / \Delta, \quad \mu^{*}=\mu^{\prime} / \Delta, \\
\alpha_{0} & =\phi_{0} / \Delta, \quad \alpha_{1}=\psi_{0} / \Delta, \quad \alpha_{2}=\phi_{1} / \Delta, \alpha_{3}=\psi_{1} / \Delta, \\
\beta & =\theta_{0} / \Delta, \quad \beta^{\prime}=\theta_{1} / \Delta, \\
\alpha_{0}^{\prime} & =\alpha_{1} \cdot \beta^{\prime}=\left(\psi_{0} \cdot \theta_{1}\right) / \Delta, \quad \alpha_{1}^{\prime}=\alpha_{0} \cdot \beta^{\prime}=\left(\phi_{0} \cdot \theta_{1}\right) / \Delta, \\
\alpha_{2}^{\prime} & =\alpha_{3} \cdot \beta=\left(\psi_{1} \cdot \theta_{0}\right) / \Delta, \quad \alpha_{3}^{\prime}=\alpha_{2} \cdot \beta=\left(\phi_{1} \cdot \theta_{0}\right) / \Delta .
\end{aligned}
$$

Then it is clear that among all the assertions of this lemma, only (ii)(e) needs to be checked; all others are direct consequences of the Claim, with slight modification if necessary. 
We will prove (ii)(e) for the case $i=0$ only. Obviously, that will suffice. First note that

$$
\left[\phi_{0}, \psi_{0} \cdot \theta_{1}\right]=[\phi \times \nu, \psi \times \theta] \leq[\phi, \psi] \times[\nu, \theta]=0_{A} \times 0_{A} \times 0_{A}=0_{A^{\prime}} .
$$

To prove $\alpha_{0}=\left(0_{A^{*}}: \alpha_{0}^{\prime}\right)$, we need to show that for every $\gamma \in \operatorname{Con} \mathbf{A}^{\prime}$, if $\gamma>\phi_{0}$, then $\gamma$ does not centralize $\psi_{0} \cdot \theta_{1}$ modulo $\Delta$. So suppose $\gamma>\phi_{0}$ and choose $\left\langle\langle a, b\rangle,\left\langle a^{\prime}, b^{\prime}\right\rangle\right\rangle \in \gamma$ so that $\left\langle a, a^{\prime}\right\rangle \notin \phi$. Then $\left\langle\langle a, a\rangle,\left\langle a^{\prime}, a^{\prime}\right\rangle\right\rangle \in \gamma$ since $\gamma \geq \eta_{0}$. Choose a term $t\left(x, y_{0}, \ldots, y_{n-1}\right)$ and choose $\left\langle c_{i}, d_{i}\right\rangle \in \psi$ $(i<n)$ so that $t(a, \bar{c})=t(a, \bar{d})$ and $t\left(a^{\prime}, \bar{c}\right) \neq t\left(a^{\prime}, \bar{d}\right)$. Now we have $\left\langle\langle a, a\rangle,\left\langle a^{\prime}, a^{\prime}\right\rangle\right\rangle \in \gamma,\left\langle\left\langle c_{i}, c_{i}\right\rangle,\left\langle d_{i}, c_{i}\right\rangle\right\rangle \in \psi_{0} \cdot \theta_{1}$ and

$$
\begin{aligned}
& \left\langle t\left(\langle a, a\rangle,\left\langle c_{0}, c_{0}\right\rangle, \ldots,\left\langle c_{n-1}, c_{n-1}\right\rangle\right), t\left(\langle a, a\rangle,\left\langle d_{0}, c_{0}\right\rangle, \ldots,\left\langle d_{n-1}, c_{n-1}\right\rangle\right)\right\rangle \in \Delta, \\
& \left\langle t\left(\left\langle a^{\prime}, a^{\prime}\right\rangle,\left\langle c_{0}, c_{0}\right\rangle, \ldots,\left\langle c_{n-1}, c_{n-1}\right\rangle\right),\right. \\
& \left.\quad t\left(\left\langle a^{\prime}, a^{\prime}\right\rangle,\left\langle d_{0}, c_{0}\right\rangle, \ldots,\left\langle d_{n-1}, c_{n-1}\right\rangle\right)\right\rangle \in \eta_{1}-\eta_{0} .
\end{aligned}
$$

But the last membership relation " $\in \eta_{1}-\eta_{0}$ " since $\Delta \cdot \eta_{1}=0_{A^{\prime}} \leq \eta_{0}$.

Finally, to prove $\alpha_{0}^{\prime}=\left(0_{A^{*}}: \alpha_{0}\right)$, suppose $\gamma>\psi_{0} \cdot \theta_{1}$. We want to show that $\gamma$ does not centralize $\phi_{0}$ modulo $\Delta$. Choose $\left\langle\langle a, b\rangle,\left\langle a^{\prime}, b^{\prime}\right\rangle\right\rangle \in \gamma-\psi_{0} \cdot \theta_{1}$. Then we must have $\left\langle a, a^{\prime}\right\rangle \notin \psi$ or $\left\langle b, b^{\prime}\right\rangle \notin \theta$. For the first case $\left\langle a, a^{\prime}\right\rangle \notin \psi$, choose a term $t\left(x, y_{0}, \ldots, y_{n-1}\right)$ and choose $\left\langle c_{i}, d_{i}\right\rangle \in \phi \quad(i<n)$ so that $t(a, \bar{c})=t(a, \bar{d})$ and $t\left(a^{\prime}, \bar{c}\right) \neq t\left(a^{\prime}, \bar{d}\right)$. Then we have $\left\langle\langle a, b\rangle,\left\langle a^{\prime}, b^{\prime}\right\rangle\right\rangle \in \gamma$, $\left\langle\left\langle c_{i}, c_{i}\right\rangle,\left\langle d_{i}, c_{i}\right\rangle\right\rangle \in \phi_{0}$ and

$$
\begin{aligned}
& \left\langle t\left(\langle a, b\rangle,\left\langle c_{0}, c_{0}\right\rangle, \ldots,\left\langle c_{n-1}, c_{n-1}\right\rangle\right),\right. \\
& \left.t\left(\langle a, b\rangle,\left\langle d_{0}, c_{0}\right\rangle, \ldots,\left\langle d_{n-1}, c_{n-1}\right\rangle\right)\right\rangle \in \Delta, \\
& \left\langle t\left(\left\langle a^{\prime}, b^{\prime}\right\rangle,\left\langle c_{0}, c_{0}\right\rangle, \ldots,\left\langle c_{n-1}, c_{n-1}\right\rangle\right),\right. \\
& \left.t\left(\left\langle a^{\prime}, b^{\prime}\right\rangle,\left\langle d_{0}, c_{0}\right\rangle, \ldots,\left\langle d_{n-1}, c_{n-1}\right\rangle\right)\right\rangle \notin \Delta .
\end{aligned}
$$

For the second case $\left\langle b, b^{\prime}\right\rangle \notin \theta$, choose a term $t\left(x, y_{0}, \ldots, y_{n-1}\right)$ and choose $\left\langle c_{i}, d_{i}\right\rangle \in \nu \quad(i<n)$ so that $t(b, \bar{c})=t(b, \bar{d})$ and $t\left(b^{\prime}, \bar{c}\right) \neq t\left(b^{\prime}, \bar{d}\right)$. Then we have $\left\langle\langle a, b\rangle,\left\langle a^{\prime}, b^{\prime}\right\rangle\right\rangle \in \gamma,\left\langle\left\langle c_{i}, c_{i}\right\rangle,\left\langle c_{i}, d_{i}\right\rangle\right\rangle \in \phi_{0}$ and

$$
\begin{array}{r}
\left\langle t\left(\langle a, b\rangle,\left\langle c_{0}, c_{0}\right\rangle, \ldots,\left\langle c_{n-1}, c_{n-1}\right\rangle\right),\right. \\
\left.t\left(\langle a, b\rangle,\left\langle c_{0}, d_{0}\right\rangle, \ldots,\left\langle c_{n-1}, d_{n-1}\right\rangle\right)\right\rangle \in \Delta, \\
\left\langle t\left(\left\langle a^{\prime}, b^{\prime}\right\rangle,\left\langle c_{0}, c_{0}\right\rangle, \ldots,\left\langle c_{n-1}, c_{n-1}\right\rangle\right),\right. \\
\left.t\left(\left\langle a^{\prime}, b^{\prime}\right\rangle,\left\langle c_{0}, d_{0}\right\rangle, \ldots,\left\langle c_{n-1}, d_{n-1}\right\rangle\right)\right\rangle \notin \Delta .
\end{array}
$$

This completes the proof.

Theorem 8. Let A be a finite subdirectly irreducible member of $\mathscr{V}$ with an abelian monolith $\mu$, and let $\nu=\left(0_{A}: \mu\right)$. Then

(i) $\operatorname{typ}\left\{\nu, 1_{A}\right\} \subseteq\{3\}$.

(ii) $\mathbf{I}\left[\nu, 1_{A}\right]$ is a linear lattice.

(iii) If $\alpha \in$ Con $\mathbf{A}$ is solvable, then $\alpha \leq \nu$.

(iv) $\nu$ is solvable, and $\theta \stackrel{\text { def }}{=}\left(0_{A}: \nu\right)$ is the largest abelian congruence of $\mathbf{A}$.

Proof. Let $\theta=\left(0_{A}: \nu\right)$. Then $\theta \geq \mu$ as $\mu$ centralizes $\nu$. $\theta$ centralizes $\mu$ since $\theta$ centralizes $\nu$ which is above $\mu$. Thus $\theta \leq \nu$. Note that $\left(0_{A}: \theta\right) \geq$ $\nu$ since $\nu$ centralizes $\theta$. On the other hand, $\left(0_{A}: \theta\right) \leq \nu$ since $\left(0_{A}: \theta\right)$ centralizes $\mu$ (as $\left(0_{A}: \theta\right)$ centralizes $\left.\theta \geq \mu\right)$. So we have $\theta \leq \nu=\left(0_{A}: \theta\right)$. 
Now, by Lemma 6, the statements (i) and (ii) follow. To prove (iii), suppose that $\alpha \in$ Con $\mathbf{A}$ is solvable and $\alpha \notin \nu$. To get a contradiction, observe that $\mathbf{I}[\nu, \nu \vee \alpha]$ is a nontrivial interval that projects down to $\mathbf{I}[\nu \wedge \alpha, \alpha]$. This implies that $\operatorname{typ}\left\{0_{A}, \alpha\right\}$ contains 3 , which contradicts the supposition that $\alpha$ is solvable. (See (6.2) and (7.2) of [7].)

To prove (iv), let $\theta=\left(0_{A}: \nu\right)$. $\theta$ must be abelian since $\theta$ centralizes $\nu$ which is $\geq \theta$. Suppose that there exists an abelian congruence $\theta^{\prime}$ which is not $\leq \theta$. Then $\theta^{\prime} \leq\left(0_{A}: \theta^{\prime}\right)<\nu$. Then, by Lemma 6 , we have $3 \in$ $\operatorname{typ}\left\{\theta^{\prime}, \nu\right\}$, which implies that $\nu$ is not solvable. Now that $\theta$ is abelian (and hence solvable), type $\{\theta, \nu\}$ should contain 3 . We will get a contradiction by semantically embedding the class of all finite Boolean triples into $\mathrm{V}(\mathbf{A})_{\text {fin }}$. By Lemma 7, we can assume wlog that Con $\mathbf{A}$ contains congruences $\alpha_{0}, \alpha_{1}$, and $\beta$ such that $\alpha_{0} \wedge \alpha_{1}=\beta>\theta$ and $\alpha_{i} \nsubseteq \alpha_{1-i}(i<2)$. Further, we can assume that $3 \in \operatorname{typ}\left\{\beta, \alpha_{0}\right\} \cap \operatorname{typ}\left\{\beta, \alpha_{1}\right\} \cap \operatorname{typ}\{\theta, \beta\}$. For each $i<3$, choose $\delta_{i}, \gamma_{i} \in$ Con $\mathbf{A}$ so that $\beta \leq \delta_{i} \prec \gamma_{i} \leq \alpha_{i} \quad(i<2), \theta \leq \delta_{2} \prec \gamma_{2} \leq \beta$ and $\operatorname{typ}\left(\delta_{i}, \gamma_{i}\right)=3$ $(i<3)$. Figure 3 shows a part of Con $\mathbf{A}$. Let $U_{i} \stackrel{\text { def }}{=}\left\{0_{i}, 1_{i}\right\} \in M_{\mathbf{A}}\left(\delta_{i}, \gamma_{i}\right)$ for each $i<3$. Choose meet $(x, y) \in \operatorname{Pol}_{2} \mathbf{A}$ so that meet $\left.\right|_{U_{2}}$ is the Boolean meet operation of $\left.\mathbf{A}\right|_{U_{2}}$. Let a finite Boolean triple $\mathbf{B} \stackrel{\text { def }}{=}\left\langle B, \leq, B_{0}, B_{1}\right\rangle$ be given. Wlog $B$ is the power set of some finite set $X$ and $\leq$ is just the set theoretic inclusion relation. Let $\mathbf{D}$ be the subalgebra of $\mathbf{A}^{\bar{X}}$ with universe $\left\{f \in A^{X} \mid f^{-1}\left(a / \gamma_{i}\right) \in B_{i}\right.$ for every $\left.a \in A, i<2\right\}$. Then $\mathbf{D}$ is a $\beta$-closed diagonal subdirect power of $\mathbf{A}$. Fix $\langle 0,1\rangle \in \mu-0_{A}$, and choose $\lambda_{i} \in \mathbf{P o l}_{1} \mathbf{A}$ so that $\left\langle\lambda_{i}\left(0_{i}\right), \lambda_{i}\left(1_{i}\right)\right\rangle=\langle 0,1\rangle$ for each $i<3$. Now we are done with the proof by the following observations:

$$
\begin{aligned}
& f \in D(\{0,1\}) \Leftrightarrow\left(\exists g \in D\left(U_{2}\right)\right)\left(\lambda_{2}(g)=f\right), \\
& f^{-1}(1) \subseteq f^{\prime-1}(1) \Leftrightarrow\left(\exists g, g^{\prime} \in D\left(U_{2}\right)\right) \\
&\left(\lambda_{2}(g)=f \& \lambda_{2}\left(g^{\prime}\right)=f^{\prime} \& \operatorname{meet}\left(g, g^{\prime}\right)=g\right), \\
& f^{-1}(1) \in B_{i} \Leftrightarrow\left(\exists g \in D\left(U_{1-i}\right)\right)\left(\lambda_{1-i}(g)=f\right) \quad \text { for each } i<2 .
\end{aligned}
$$

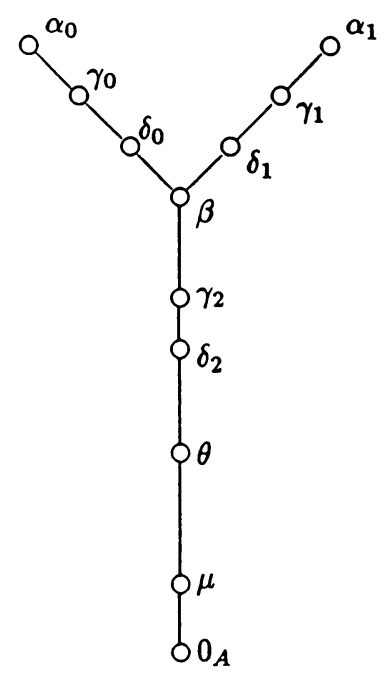

FIGURE 3 
Theorem 9. Let $\mathbf{A}$ be a finite subdirectly irreducible member of $\mathscr{V}$ with a monolith $\mu$. Then $\left(0_{A}: \mu\right)$ is abelian. Thus $\left(0_{A}: \mu\right)=\left(0_{A}:\left(0_{A}: \mu\right)\right)$ is the largest abelian congruence of $\mathbf{A}$.

Proof. Let $\mathbf{A} \in \mathscr{V}_{\mathrm{SI} \text {, fin }}$ and $\mu \in$ Con $\mathbf{A}$ be given as in the hypothesis. If $\mu$ is not abelian, then the conclusion holds trivially. So let us assume that $\mu$ is abelian. Suppose that $\left(0_{A}: \mu\right) \stackrel{\text { def }}{=} \nu$ is not abelian. Then $\left(0_{A}: \nu\right) \stackrel{\text { def }}{=} \theta<\nu$. We will get a contradiction. By Theorem $8(\mathrm{iv})$, we have

(19) if $\langle a, b\rangle \in 1_{A}-\theta$, then $\operatorname{Cg}^{\mathbf{A}}(a, b)$ is a nonabelian congruence.

By Lemma 7, we can assume wlog that Con $\mathbf{A}$ contains congruences $\alpha_{0}, \alpha_{1}$, and $\beta$ such that $\alpha_{0} \wedge \alpha_{1}=\beta>\theta, \alpha_{i} \nsubseteq \alpha_{1-i}(i<2), \alpha_{i}=\left(0_{A}:\left(0_{A}: \alpha_{i}\right)\right) \quad(i<$ 2) and $\beta=\left(0_{A}:\left(0_{A}: \beta\right)\right)$. Let $\mathbf{B} \stackrel{\text { def }}{=}\left\langle B, \leq, B_{0}, B_{1}\right\rangle$ be a finite Boolean triple. Wlog $B$ is the power set of some finite set $X$. We are going to semantically embed $\mathbf{B}$ into a finite member of $\mathrm{V}(\mathbf{A})$, namely $\mathbf{D} \leq_{\text {diag }} \mathbf{A}^{X}$ with $D=\{f \in$ $A^{X} \mid f^{-1}\left(a / \alpha_{i}\right) \in B_{i}$, for every $\left.a \in A, i<2\right\}$ as in the proof of the preceding theorem.

Fix $\left\langle c_{0}, c_{1}\right\rangle \in \beta-\theta,\langle 0,1\rangle \in \mu-0_{A}$. We first claim that $D\left(c_{0} / \theta \cup c_{1} / \theta\right)$ is first order definable in $\mathbf{D}$. To prove the claim, let $\operatorname{Bet}(x, y)$ and $\operatorname{Nu}(x, y)$ be first order formulas such that whenever $\mathbf{D} \leq_{\text {diag }} \mathbf{A}^{X}, f, g \in D$,

$$
\mathbf{D} \vDash \operatorname{Bet}(f, g) \Leftrightarrow\langle f, g\rangle \in D(\beta), \quad \mathbf{D} \vDash \operatorname{Nu}(f, g) \Leftrightarrow\langle f, g\rangle \in D(\nu) .
$$

Such formulas exist by the remark following (7). So, in particular, $D\left(c_{0} / \beta\right)$ is first order definable in $\mathbf{D}$.

Let the formulas $\operatorname{Img}(x, y, z, w), \operatorname{Red}(x, y, z, w)$, and $\operatorname{Dom}_{a, b}(x, y)$ (for each $a, b \in A$ ) be the same as in the proof of Lemma 6 except that we replace $\operatorname{Tht}(u, v)$ in $\operatorname{Red}(x, y, z, w)$ by $\operatorname{Nu}(u, v)$. Then it is easy to check that a little modification of the proof of Lemma 6 Claim 1 yields if $\mathbf{D} \leq \operatorname{diag} \mathbf{A}^{X}$ is $\beta$-closed and $f \in D, g \in D\left(c_{0} / \beta\right)$, and $b \in c_{0} / \beta$, then

(i) $\mathbf{D} \vDash \operatorname{Dom}_{0, b}(f, g) \Rightarrow \llbracket f \notin \hat{0} \rrbracket \subseteq \llbracket\langle g, \hat{b}\rangle \neq \theta \rrbracket$.

(ii) If $x \in X, \llbracket f \neq 0, \rrbracket=\{x\}, f(x) \in 0 / \mu$, and $g(x) \notin b / \theta$, then $\mathbf{D} \vDash \operatorname{Dom}_{0, b}(f, g)$.

Note that, in proving (ii), we may need to use (6), (19) and the fact that $D$ is $\beta$-closed. Now the claim is proved by the following observation: for all $f \in D$,

$$
\begin{aligned}
f \in D\left(c_{0} / \theta \cup c_{1} / \theta\right) \Leftrightarrow & f \in D\left(c_{0} / \beta\right) \& \\
& \neg(\exists g \neq \hat{0})\left(\operatorname{Dom}_{0, c_{0}}(g, f) \& \operatorname{Dom}_{0, c_{1}}(g, f)\right) .
\end{aligned}
$$

Next, we want to show that $D(\{0,1\})$ is first order definable in $\mathbf{D}$. To this end, find $s \in \mathrm{Pol}_{2} \mathrm{~A}$ and $a \in A$ so that

$$
d_{0} \stackrel{\text { def }}{=} p\left(s\left(c_{0}, c_{0}\right), s\left(c_{0}, c_{1}\right), a\right) \neq p\left(s\left(c_{1}, c_{0}\right), s\left(c_{1}, c_{1}\right), a\right) \stackrel{\text { def }}{=} d_{1},
$$

where $p$ is a Maltsev term for $\mathbf{A}$ as in (5). Such $s$ and $a$ exists by (19). Choose $\lambda \in \mathrm{Pol}_{1} \mathbf{A}$ so that $\left\langle\lambda\left(d_{0}\right), \lambda\left(d_{1}\right)\right\rangle=\langle 0,1\rangle$. Then, since the unary polynomial $q(x) \stackrel{\text { def }}{=} p\left(s\left(x, c_{0}\right), s\left(x, c_{1}\right), a\right)$ is constant on $\theta$-classes, it is clear that

$$
f \in D(\{0,1\}) \Leftrightarrow\left(\exists g \in D\left(c_{0} / \theta \cup c_{1} / \theta\right)\right)(\lambda(q(g))=f) .
$$

Let $\operatorname{Min}(x)$ be a first order formula such that, whenever $\mathbf{D} \leq_{\operatorname{diag}} \mathbf{A}^{X}$ and $f \in D$, we have 


$$
\begin{array}{r}
\operatorname{Min}(f) \Leftrightarrow\left(\exists g \in D\left(c_{0} / \theta \cup c_{1} / \theta\right)\right)\left[\left(\lambda(q(g))=f \& f \neq \hat{0} \& \forall h, h^{\prime} \in D(\{0,1\})\right.\right. \\
\left.\left(\left(\operatorname{Dom}_{0, c_{0}}(h, g) \& \operatorname{Dom}_{0, c_{0}}\left(h^{\prime}, g\right) \& h \neq \hat{0} \neq h^{\prime}\right) \Rightarrow h=h^{\prime}\right)\right] .
\end{array}
$$

Note that, for $f \in D(\{0,1\}), \mathbf{D} \vDash \operatorname{Min}(f) \Leftrightarrow f^{-1}(1)$ is a singleton.

If we let $\Phi: D(\{0,1\}) \rightarrow B$ be given by $\Phi(f)=f^{-1}(1)$, then it is easily seen that $\Phi$ is bijective. " $\leq$ " on $B$ is recovered by

$$
\begin{aligned}
& \Phi(f) \subseteq \Phi\left(f^{\prime}\right) \Leftrightarrow\left(\exists g, g^{\prime} \in D\left(c_{0} / \theta \cup c_{1} / \theta\right)\right) \\
& \left(\lambda(q(g))=f \& \lambda\left(q\left(g^{\prime}\right)\right)=f^{\prime} \&\right. \\
& \left.\quad \forall h \in D(\{0,1\})\left(\operatorname{Min}(h) \& \operatorname{Dom}_{0, c_{0}}(h, g) \Rightarrow \operatorname{Dom}_{0, c_{0}}\left(h, g^{\prime}\right)\right)\right) .
\end{aligned}
$$

It remains to recover $B_{i} \quad(i<2)$. Obviously, it would be sufficient to do this for $i=0$. Pick any $\left\langle 0^{1}, 1^{1}\right\rangle \in \alpha_{1}-\beta$. Then $D\left(0^{1} / \alpha_{1}\right)$ is first order definable by the remark following (7). By (7) again, we can find $\langle u, v\rangle \in\left(0_{A}: \beta\right)$ and $a \in A$ so that

$$
d_{0}^{1} \stackrel{\text { def }}{=} p\left(s\left(0^{1}, u\right), s\left(0^{1}, v\right), a\right) \neq p\left(s\left(1^{1}, u\right), s\left(1^{1}, v\right), a\right) \neq d_{1}^{1} .
$$

Choose $\lambda^{1} \in \operatorname{Pol}_{1} \mathbf{A}$ so that $\left\langle\lambda^{1}\left(d_{0}^{1}\right), \lambda^{1}\left(d_{1}^{1}\right)\right\rangle=\langle 0,1\rangle$. Then, since the unary polynomial $q^{1}(x) \stackrel{\text { def }}{=} p(s(x, u), s(x, v), a)$ is constant on $\beta$-classes, it is clear that

$$
\Phi(f) \in B_{0} \Leftrightarrow\left(\exists g \in D\left(0^{1} / \alpha_{1}\right)\right)\left(\lambda^{1}\left(q^{1}(g)\right)=f\right) .
$$

This completes the proof.

Theorem 10. Let $\mathscr{V}$ be a finitely decidable congruence modular variety. Then,

(i) Each locally finite algebra in $\mathscr{V}$ satisfies the commutator law $x \cdot[y, y] \approx$ $[x \cdot y, y]$.

(ii) Every finitely generated subvariety of $\mathscr{V}$ has a finite bound on the cardinalities of its subdirectly irreducible members.

(iii) Solvable congruences in any locally finite member of $\mathscr{V}$ are abelian. Thus a finite algebra in $\mathscr{V}$ always has the largest abelian congruence.

(iv) Each locally finite member of $\mathscr{V}$ is centerless modulo its center, i.e., the second center coincides with the first center.

Proof. To prove (i), first note that the commutator law $x \cdot[y, y] \approx[x \cdot y, y]$, which will be called ( $\mathrm{C} 1)$, is a local property. Thus, if we assume that (i) is false, then by $[6,(8.1)]$, there exists a finite algebra $\mathbf{A} \in \mathscr{V}$ and $\theta, \psi \in \operatorname{Con} \mathbf{A}$ such that $\theta \leq[\psi, \psi]$ and $\theta>[\theta, \psi]$. Wlog we may assume that $[\theta, \psi]=0_{A}$. We will prove that the existence of such an algebra $\mathbf{A}$ leads to a contradiction by induction on $|A|$. By Theorem 8 and Theorem 9, we may exclude the possibility that $\mathbf{A}$ is subdirectly irreducible. So we can choose a nontrivial subdirect embedding $\mathbf{A} \leq_{\text {sd }} \mathbf{A}_{0} \times \mathbf{A}_{1}$. Let $\pi_{i}: A \rightarrow A_{i}$ be the projection map and let $\eta_{i}=$ kernel of $\pi_{i}$ for each $i<2$. Since $\theta>0_{A}=\eta_{0} \cdot \eta_{1}$, we must have either $\theta \leq \eta_{0}$ or $\theta \leq \eta_{1}$. Consider the case $\theta \leq \eta_{0}$. If we let $\theta^{\prime}=\pi_{0}\left(\theta+\eta_{0}\right)$ and $\psi^{\prime}=\pi_{0}\left(\psi+\eta_{0}\right)$, then we get

$$
\begin{aligned}
{\left[\theta^{\prime}, \psi^{\prime}\right] } & =\left[\pi_{0}\left(\theta+\eta_{0}\right), \pi_{0}\left(\psi+\eta_{0}\right)\right]=\pi_{0}\left([\theta, \psi]+\eta_{0}\right)=\pi_{0}\left(\eta_{0}\right)=0_{A_{0}}<\theta^{\prime} \\
& =\pi_{0}\left(\theta+\eta_{0}\right) \leq \pi_{0}\left([\psi, \psi]+\eta_{0}\right)=\left[\pi_{0}\left(\psi+\eta_{0}\right), \pi_{0}\left(\psi+\eta_{0}\right)\right]=\left[\psi^{\prime}, \psi^{\prime}\right] .
\end{aligned}
$$


So we have go a failure of the law $x \cdot[y, y] \approx[x \cdot y, y]$ in the algebra $\mathbf{A}_{0}$ which has less cardinality than $\mathbf{A}$. We are done with the case $\theta \leq \eta_{0}$. The other remaining case $\theta \not \eta_{1}$ can be handled similarly. This completes the proof of (i).

(ii) is the direct consequence of (i) and [6, (10.15)]. To prove (iii), let $\theta$ be a solvable congruence in a locally finite algebra $\mathbf{A} \in \mathscr{V}$. If $\mathbf{A}$ has a solvable congruence that is not abelian, then some finitely generated subalgebra of $\mathbf{A}$ will also have a solvable congruence that is not abelian. Thus wlog $\mathbf{A}$ is finitely generated (i.e., finite). Take a subdirect representation $\mathbf{A} \leq_{\text {sd }} \mathbf{A}_{0} \times \cdots \times \mathbf{A}_{n-1}$. Then, for each $i<n, \pi_{i}(\theta) \stackrel{\text { def }}{=} \theta^{i} \in$ Con $\mathbf{A}_{i}$ is a solvable congruence in the finite subdirectly irreducible algebra $\mathbf{A}_{i} \in \mathscr{V}$ and hence abelian. But then $\theta^{*} \stackrel{\text { def }}{=} \theta^{0} \times \cdots \times \theta^{n-1} \in \operatorname{Con} \mathbf{A}_{0} \times \cdots \times \mathbf{A}_{n-1}$ is abelian and thus $\theta \leq\left.\theta^{*}\right|_{A}$ must be abelian also.

Finally, to prove (iv), suppose that there exists a locally finite algebra $\mathbf{A}$ of $\mathscr{V}$ for which the first center $Z_{1}$ is strictly less than the second center $Z_{2}$. We are going to get a contradiction. By supposition, we have $\mathrm{e}\left[1_{A}, 1_{A}\right] \geq$ $\left[Z_{2}, 1_{A}\right] \stackrel{\text { def }}{=} \theta \leq Z_{1}$. Then $\left[\theta, 1_{A}\right]=\left[\theta \cdot 1_{A}, 1_{A}\right]=\theta \cdot\left[1_{A}, 1_{A}\right]=\theta$ by $(\mathrm{C} 1)$. But $\left[\theta, 1_{A}\right] \leq\left[Z_{1}, 1_{A}\right]=0_{A}$. Hence $\theta=\left[\theta, 1_{A}\right]=0_{A}$. We have shown that $Z_{2}$ centralizes $1_{A}$, which contradicts our supposition.

McKenzie and Valeriote showed that the property $Z_{2}=Z_{1}$ holds for finite algebras in decidable varieties [17]. The statement (iv) above is close to this. It replaces "finite" by "locally finite" and "decidable" by "finitely decidable or decidable" with the additional assumption of congruence modularity.

\section{Discussion}

Throughout this section, we let $\mathscr{V}$ be a finitely generated congruence modular variety of finite type satisfying all the necessary conditions for finite decidability stated in this paper together with congruence permutability (which has been shown to be necessary in [13]).

Recently Albert [1] showed that $\mathscr{V}$ is finitely decidable if it satisfies the following two more conditions:

(i) for each finite subdirectly irreducible algebra in $\mathscr{V}$, if the monolith is abelian, then $\mu=\left(0_{A}: \mu\right)$,

(ii) the abelian subvariety of $\mathscr{V}$ is finitely decidable.

In fact the condition (ii) above is easily seen to be necessary for finite decidability of $\mathscr{V}$ since an algebra in $\mathscr{V}$ is abelian iff it satisfies a first order sentence saying that a Maltsev term for $\mathscr{V}$ commutes with each basic operation.

Several months after Albert's result, Idziak [11] claims that $\mathscr{V}$ is finitely decidable if

(i) for each finite subdirectly irreducible algebra in $\mathscr{V}$, the centralizer of the monolith is comparable to every congruence of $\mathbf{A}$,

(iii) for each finite subdirectly irreducible algebra $\mathbf{A}$ in $\mathscr{V}$ having a nonabelian monolith, the variety of $R(\mathscr{W}, n)$-modules is finitely decidable, where $n$ is the cardinality of $A$ and $\mathscr{W}$ is the subvariety of $\mathscr{V}$ generated by all subdirectly irreducible algebras $\mathbf{B}$ of $\mathscr{V}$ such that some homomorphic image of $\mathbf{B}$ is isomorphic to $\mathbf{A}$.

For the notation $R(\mathscr{W}, n)$ in (ii), the readers are referred to [6]. Condition (i) above has been shown to be necessary by Idziak and Valeriote [12]. So 
now the characterization problem of finite decidability for finitely generated congruence modular variety of finite type has been solved modulo the problem of characterizing finite decidability of module varieties. The question whether Albert's condition (ii) and Idziak's condition (i) are sufficient for the finite decidability of $\mathscr{V}$ is still open.

The result presented in this paper is a part of the Ph.D. thesis of the author [14]. The author wishes to thank his advisor Professor R. McKenzie for his very helpful suggestions and the constant encouragement throughout the period of this research.

\section{REFERENCES}

1. M. Albert, A sufficient condition for finite decidability, Trans. Amer. Math. Soc. (to appear).

2. S. Burris and R. McKenzie, Decidability and Boolean representations, Mem. Amer. Math. Soc. 32 (1981).

3. S. Burris, R. McKenzie, and M. Valeriote, Decidable discriminator varieties form unary varieties, J. Symbolic Logic 56 (1991), 1355-1368.

4. S. Burris and H. Sankappanavar, A course in universal algebra, Graduate Texts in Math., Springer-Verlag, New York, 1981.

5. J. Ershov, I. Lavrov, A. Tatmanov, and M. Taitslin, Elementary theories, Russian Math. Surveys 20 (1965), 35-105.

6. R. Freese and R. McKenzie, Commutator theory for congruence modular varieties, London Math. Soc. Lecture Note Ser., no. 125, 1987.

7. D. Hobby and R. McKenzie, The structure of finite algebras, Contemp. Math., vol. 76, Amer. Math. Soc., Providence, R.I., 1988.

8. P. Idziak, Reduced sub-powers and the decision problem for finite algebras in arithmetical varieties, Algebra Universalis 25 (1988), 365-383.

9. __ Varieties with decidable finite algebras. I: Linearity, Algebra Universalis 26 (1989), 234-246.

10. _ (1989), 247-256.

11. _ Characterization of finitely decidable congruence modular varieties, preprint, 1992.

12. P. Idziak and M. Valeriote, $A$ property of the solvable radical in finitely decidable varieties, preprint.

13. J. Jeong, Finitary decidability implies congruence permutability for congruence modular varieties, Algebra Universalis 29 (1992), 441-448.

14. __ On finitely decidable varieties, Ph. D. thesis, Univ. of California, Berkeley, 1991.

15. R. McKenzie, Narrowness implies uniformity, Algebra Universalis 15 (1982), 67-85.

16. R. McKenzie, G. McNulty, and W. Taylor, Algebras, lattices, varieties, vol. 1, Wadsworth \& Brooks/Cole, Monterey, Calif., 1987.

17. R. McKenzie and M. Valeriote, The structure of decidable locally finite varieties, Progr. Math., no. 79, Birkhäuser, Boston, Mass., 1989.

18. M. Rabin, A simple method for undecidability proofs and some applications, Logic, Methodology Philos. Sci., North-Holland, Amsterdam, 1965, pp. 58-68.

19. M. Valeriote, Decidable unary varieties, Algebra Universalis 27 (1987), 1-20.

20. M. Valeriote and R. Willard, Some properties of finitely decidable varieties, Internat. J. Algebra Comput. 2 (1992), 89-101.

21. R. Willard, Manuscript, 1990.

22. A. Zamyatin, A prevariety of semigroups whose elementary theory is solvable, Algebra and Logic 12 (1973), 233-241. 
23. __ Varieties of associative rings whose elementary theory is decidable, Soviet Math. Dokl. 17 (1976), 996-999.

24. Logic 17 (1978), 13-17.

25. __ Prevarieties of associative rings whose elementary theory is decidable, Sibirsk. Math. Zh. 19 (1978), 890-901.

Department of Pure Mathematics, University of Waterloo, Waterloo, Ontario, Canada N2L 3G1

E-mail address: jjeong@math.waterloo.edu 